\title{
Pattern Recognition Receptors and Infectious Diseases
}

\author{
Ardi Liaunardy Jopeace ${ }^{1}$, Chris B. Howard ${ }^{1}$, Ben L. Murton ${ }^{1}$, \\ Alexander D. Edwards² and Tom P. Monie ${ }^{1}$ \\ ${ }^{1}$ Department of Biochemistry, University of Cambridge and \\ ${ }^{2}$ School of Pharmacy, University of Reading \\ United Kingdom
}

\section{Introduction}

\subsection{The innate immune system}

Our bodies are under constant attack from pathogens. Despite this continual bombardment, under normal circumstances we remain healthy for most of our lives. This protection against infectious and harmful agents is provided by our immune system. The immune system can be broken into two elements: adaptive immunity and innate immunity. Adaptive immunity is a specific response targeted against particular pathogens through, for example, cytotoxic $\mathrm{T}$ cells and antibody production. The adaptive immune system has the potential to raise a defence against any invading pathogen. However, this is a relatively slow and energy expensive process. Innate immunity in contrast provides a non-specific response against any pathogen via a variety of components and processes. These include: barrier functions, complement, natural killer (NK) cells, antimicrobial peptides, mucosal secretions, pattern recognition receptors (PRRs) and the commensal micro-organisms. Innate immunity is responsible for clearing the majority of pathogen exposures that would result in infection before the adaptive system is even involved. This chapter will focus upon the role of one particular arm of the innate immune response to infectious diseases - Pattern Recognition Receptors. It will broadly address the mechanisms by which PRRs recognise the pathogens, the effects this has and the types if response it has. It will also bring in examples of evasion strategies used by pathogens to avoid detection and touch on the impact of polymorphisms in the receptors. Finally we will discuss the role of PRRs in a key defence against infectious diseases, vaccination.

\subsection{Targets for innate immune recognition by PRRs}

PRRs are protein molecules encoded in the genome and not subject to rearrangement or variation during the lifetime of an individual. PRRs function as molecular sensors of infection and are predominantly found on critical immune cells such as macrophages and dendritic cells (DC). However, other cell types likely to come into contact with pathogens, for example epithelial cells, also express subsets of these receptors. Given the absence of functional rearrangement how do PRRs recognise pathogens from diverse families possessing such diverse biology and patterns of infection? 
Firstly, the innate immune system is designed to recognise biological components that are common to many pathogens. These are known as pathogen-associated molecular patterns (PAMPs). This means, for example, that a receptor is able to respond to all bacteria that have common components in their cell walls rather than a specific protein that is found on only one type of micro-organism (Table 1).

Secondly, there are a number of families of receptors, and many receptors in each family (Section 2, Table 1). This limited diversity allows the innate immune system to respond, not only to different PAMPs, but also PAMPs found in either the extracellular space or an intracellular environment. By having multiple sites for detection of diverse targets, it is unlikely that any given pathogen will be able to evade all of the levels of detection.

Thirdly, and most importantly, the receptors are able to mount a coordinated response to pathogen infection because of extensive cross-talk and communication between the different signalling pathways. This again minimises the possibility of a pathogen being able to evade the innate immune response. Overall, the innate immune response is primarily designed to induce inflammation at the site of infection, recruit inflammatory cells and mediators and begin to potentiate the adaptive immune system. The coordinated nature of the innate response ensures that any response initiated is robust enough to meet the threat.

\begin{tabular}{|c|c|c|c|}
\hline $\begin{array}{l}\text { Stimulatory Pathogen } \\
\text { Associated Molecular } \\
\text { Pattern (PAMP) }\end{array}$ & $\begin{array}{l}\text { Pattern Recognition } \\
\text { Receptor (PRR) }\end{array}$ & $\begin{array}{c}\text { Signalling } \\
\text { Adapter Protein }\end{array}$ & $\begin{array}{c}\text { Transcriptional or } \\
\text { Cellular Pathway } \\
\text { Activated }\end{array}$ \\
\hline \multicolumn{4}{|c|}{ Toll-like receptors (TLRs) } \\
\hline $\begin{array}{l}\text { Bacterial cell wall } \\
\text { components }\end{array}$ & $\begin{array}{c}\text { TLR2 } \\
\text { homo/heterodimers }\end{array}$ & MyD88 & NFKB / AP1 \\
\hline LPS & $\begin{array}{c}\text { TLR4 (plasma } \\
\text { membrane) }\end{array}$ & MyD88 & NFKB / AP1 \\
\hline LPS & TLR4 (endosome) & TRIF & IRF3 / NFKB / AP1 \\
\hline Flagellin & TLR5 & MyD88 & NFKB / AP1 \\
\hline dsRNA & TLR3 & TRIF & IRF3 / NFKB / AP1 \\
\hline ssRNA & TLR7 & MyD88 & IRF7 / NFKB \\
\hline \multicolumn{4}{|c|}{ Nod-like receptors (NLRs) } \\
\hline iE-DAP & NOD1 & RIP2 & NFKB \\
\hline MDP & NOD2 & RIP2/CARD9 & NFKB / AP1 \\
\hline $\begin{array}{l}\text { e.g. Pore-forming } \\
\text { toxins, nucleic acid }\end{array}$ & NLRP3 & ASC & Caspase- 1 activation \\
\hline \multicolumn{4}{|c|}{ Retinoic acid-inducible gene I-like receptors (RLRs) } \\
\hline dsRNA & RIG-I & MAVS & IRF3 / AP1 / NFKB \\
\hline \multicolumn{4}{|c|}{ C-type lectin receptors (CLRs) } \\
\hline$\beta$-glucans & Dectin-1 & Syk & NFKB \\
\hline
\end{tabular}

Table 1. Pattern Recognition Receptor (PRR) Activation and Outputs. The activation and signalling of PRRs is a complex, multi-factorial process. Activatory ligands (blue column) are recognised by specific PRRs (pink column). This leads to the recruitment of adaptor signalling proteins (lilac column) and the activation of intracellular signalling cascades. The net result is the up-regulation of transcriptional activators or specific cellular processing events (green column). 


\subsection{Innate immune signalling overview}

PRR activation and signalling is a complex multifactorial process that results in remarkably similar outcomes (Figure 1). For example: upregulation of NFкB (Nuclear Factor kappa B) and IRF (interferon (IFN) regulatory factor) family transcription factors; stimulation of the stress kinase pathways (e.g. mitogen-activated protein kinases (MAPK)); and activation of caspase-1 (Figure 1). Ultimately this results in up-regulation of pro-inflammatory cytokines, chemokines and anti-viral proteins. PRRs are activated by PAMPs. Endogenous molecules, such as ATP and heat shock proteins, can also act as ligands for some PRRs. These endogenous ligands are collectively known as damage associated molecular patterns (DAMPs). PRR activation results in conformational changes in the proteins, activates intracellular signalling pathways to amplify the signal and initiates the innate response (Figure 1). Assembly of the downstream signalling complex is reliant on the involvement of specific adapter proteins to recruit signalling components and act as molecular scaffolds for complex assembly. PRRs use specific adaptors and different adaptor proteins result in the activation of different signalling pathways (see Section 2; Table 1).

\subsection{Physiological outcomes of innate immune activation}

The targets of the transcription factors produced as a result of PRR stimulation are proinflammatory effectors, the most important of which are tumour necrosis factor (TNF), interleukin (IL)-1 and IL-6. The pro-inflammatory signals modify the permeability of the vasculature around a site of infection to increase recruitment of specialised immune cells, such as monocytes and macrophages. This leads to the classic signs of infection; redness, heat, swelling and pain. At a cellular level these effectors can regulate cellular death in localised areas of infection, but also coordinate events in the whole body through the activation of the acute-phase response. The production of anti-viral type I IFNs induces apoptosis in infected cells, thereby removing the virus from the system, but also triggers resistance to viral infection in neighbouring cells and so helps restrict the spread of infection.

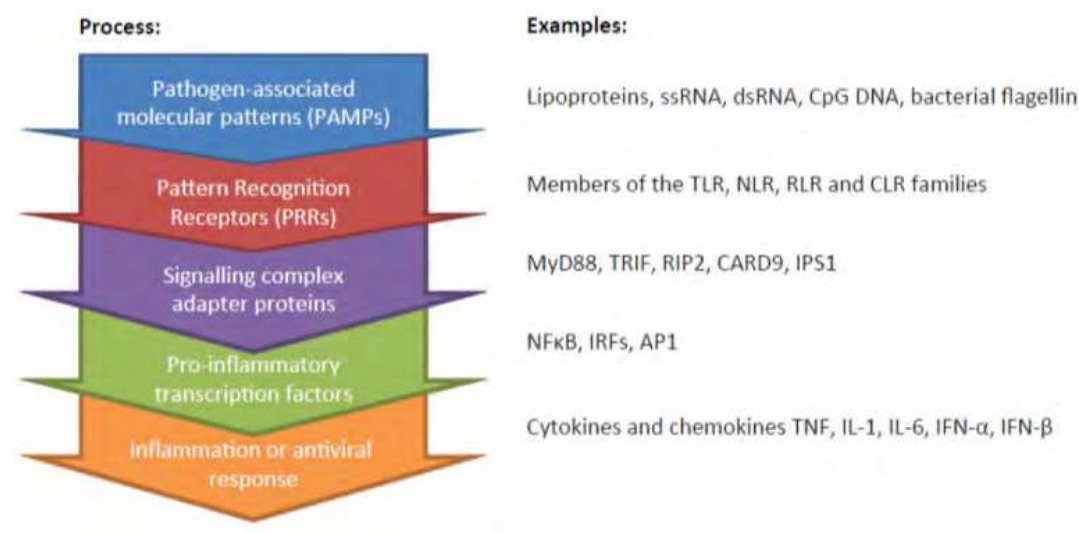

Fig. 1. Overview of innate immune signalling pathways and components. Activation begins with recognition of a stimulatory ligand, such as a pathogen-associated molecular pattern and results in an inflammatory or antiviral response from the cell. 
The cellular and molecular changes associated with PRR activation are both complex and subtle. They create a response that can be shaped to deal with the specific nature of the infection. In macrophages many genes that modify their direct involvement in fighting infection and help repair damaged tissues are activated in response to PRR stimulation. Other cells, including monocytes, neutrophils, T-cells and B-cells can also be recruited to either directly help with the immune response or aid in the activation of the adaptive immune system.

\section{Pattern recognition receptor families}

PRRs are classified into four main families: Toll-like receptors (TLRs); Nucleotide binding leucine rich repeat (NLR) containing receptors, also known as NOD-like receptors; Retinoic acid-inducible gene I (RIG-I)-like receptors; and the C-type lectin receptors. The following sections provide brief details on the general signalling strategy of each of these families as well as specific examples of stimulatory ligands and physiological responses.

\subsection{Toll-like receptors (TLRs)}

TLRs are type 1 membrane proteins. They were the first components discovered in what we now regard as the innate immune system. TLRs use leucine-rich repeats (LRRs) to detect and bind ligand (Monie et al., 2009). LRRs have a conserved structural backbone which provides a scaffold on which variation can be built (Figure 2). Hence, LRRs from different receptors recognise a diverse range of PAMPs. In humans, TLR1, 2, 4, 5, 6 and 10 project their LRRs into the extracellular space, whereas TLR3, 7, 8 and 9 are compartmentalised to sample the contents of the endosomes.

TLRs signal in dimeric complexes after ligand binding (Figures 2 and 3). In general these are homodimeric complexes except for TLR2 which forms a heterodimer with TLR1 or TLR6. It has also been reported that TLR4 is capable of forming a signalling complex with TLR6. However, in this instance the receptor is involved in sterile inflammation and, in conjunction with CD36 responds to endogenous danger signals rather than PAMPs (Stewart et al., 2009). It is conceivable that following activation multiple dimeric TLR receptors cluster together in specific regions of the cellular membrane in order to augment signalling. Extracellular TLRs generally recognise components found on the outer surfaces of pathogens, such as lipoproteins and flagellin. Endosomal TLRs meanwhile recognise nucleic acids such as CpG-DNA, double-stranded RNA (dsRNA) and single stranded RNA (ssRNA).

The cytoplasmic Toll/IL-1 receptor (TIR) domain (Figure 2) mediates downstream signalling through adaptor recruitment. Based upon adaptor usage TLR signalling can be divided into two categories; those that signal through the protein myeloid differentiation factor 88 (MyD88) and those that don't. MyD88 is recruited to the TIR domains of an activated TLR and results in the formation of a multiprotein complex termed the myddosome (Figure 3). The myddosome contains a number of IL-1R-associated kinases (IRAKs) which direct signalling down specific pathways. Firstly, degradation of the inhibitory protein IKB (inhibitor of kappa B) releases the transcription factor $N F \kappa B$; and secondly, the MAP (mitogen activated protein) kinase pathway activates the c-fos/jun transcription factor. These signals combine to drive expression of pro-inflammatory 
cytokines from NFKB and AP1 responsive genes respectively. The endosomal TLRs use the same pathway to activate NFKB and members of the IRF-family of transcription factors that activate expression of type I interferons needed to combat viral infection.
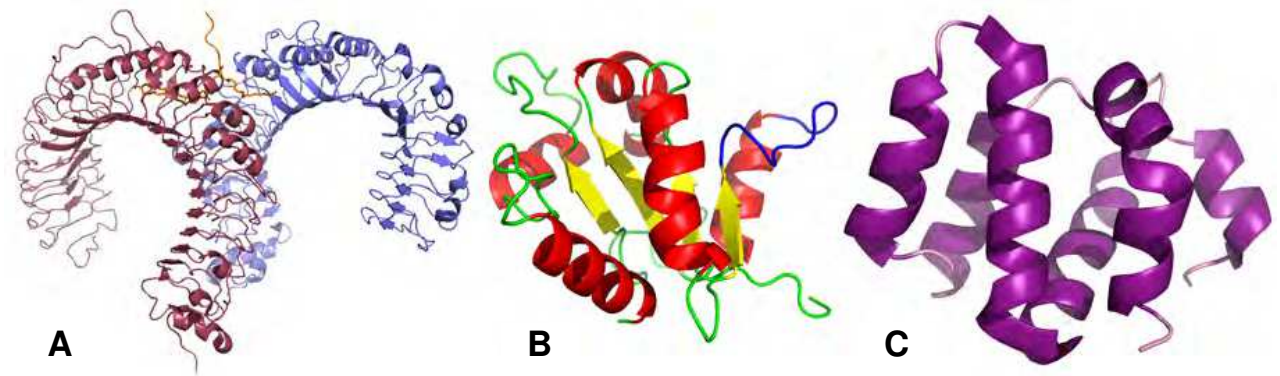

Fig. 2. Selected secondary structures of functional domains from PRRs. (A) Leucine rich repeat (LRR) containing ectodomains of TLR1 (blue) and TLR2 (purple). The synthetic ligand PAM3CYK4 (orange) stimulates heterodimerisation and formation of a signalling competent complex. (B) Toll/IL-1 receptor (TIR) domain of TLR2. The central beta-sheet core (yellow) is surrounded by five alpha helices. The BB loop, between the second betasheet and second alpha helix, is coloured blue. The BB loop is a key region of the TIR domain for downstream signalling activation and adaptor recruitment. (C) The caspase activation and recruitment domain (CARD) of the NLR family member NOD1. The CARD domain is a six-helix bundle involved in protein-protein interactions.

The MyD88-independent pathways use the adapter TIR-related adaptor protein inducing IFN $\beta$ (TRIF) in combination usually with the adaptor protein TRAM (TRIF-related adaptor molecule). This pathway is used to drive expression of IFN- $\beta$ either in response to dsRNA detection by TLR3, or TLR4 signalling from the endosome rather than the plasma membrane. There is however significant cross-talk between the pathways. Adaptor proteins have recently been shown to be important in the susceptibility to infectious disease. This is exemplified by the adaptor Mal (MyD88-adaptor like), which is involved in recruitment of MyD88 to the TIR domain of TLR2 and TLR4. In this instance heterozygotic carriage of a single nucleotide polymorphism that results in the amino acid change serine to leucine at residue one-hundred-and-eighty in the TIR domain appears to be protective against the development of sepsis, invasive pneumococcal disease, bacteremia, malaria and tuberculosis (Ferwerda et al., 2009a; Khor et al., 2007)

\subsection{Nucleotide binding leucine rich repeat containing receptors (NLRs)}

The NLRs are a large family of cytoplasmic PRRs, of which there are at least 23 members. They share a characteristic domain organisation comprising of an $\mathrm{N}$-terminal protein interaction domain, a central nucleotide binding region and C-terminal leucine-rich repeats (Figure 2). Currently the precise method of receptor activation by all the different ligands remains to be elucidated for the NLR family. There are two main groups in the NLR family based on the nature of their N-terminal domain. These are the NLRC sub-family who possess caspase activation and recruitment domain (CARDs) (Figure 2), and the Pyrin domain containing NLRP sub-family. 


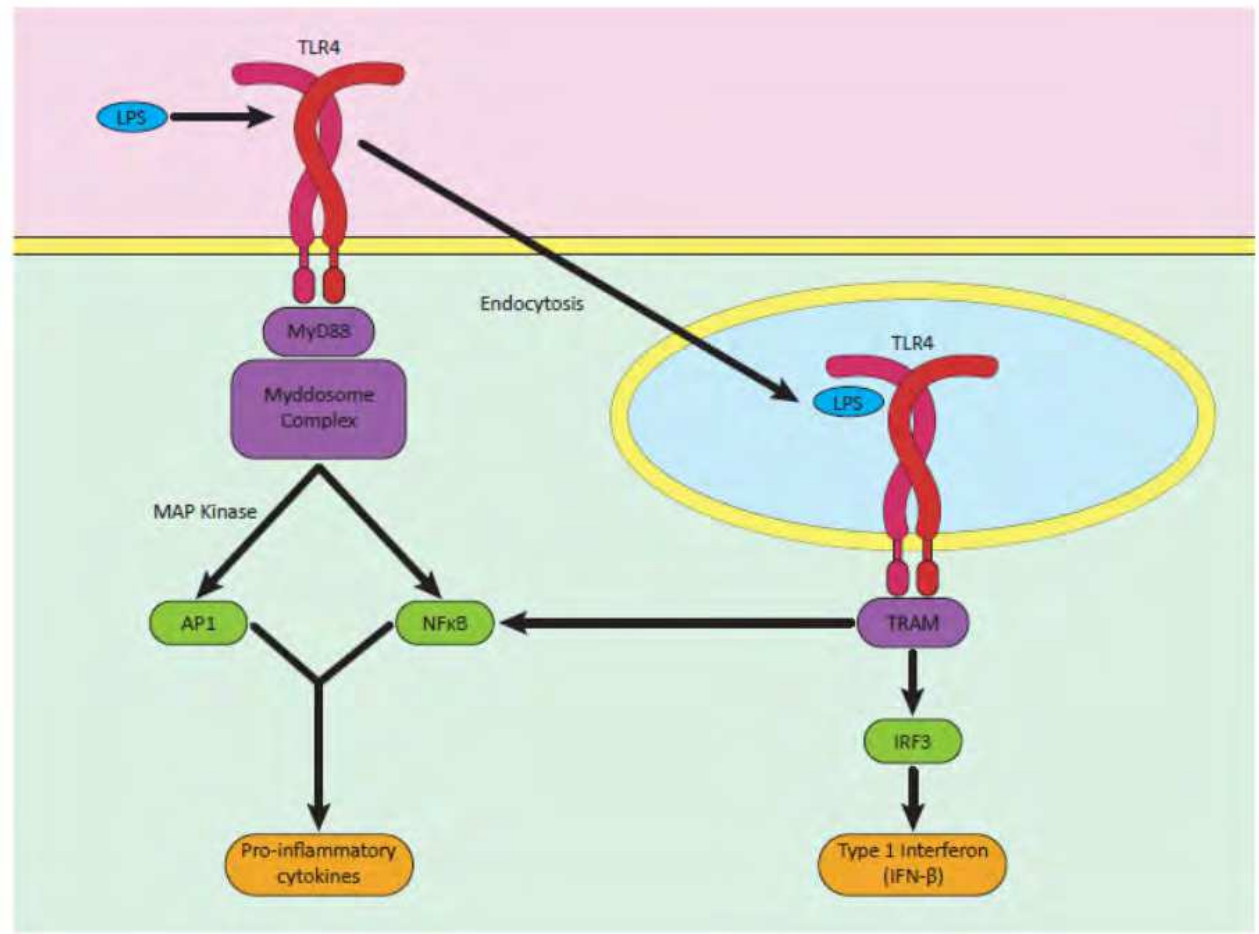

Fig. 3. Simplified schematic of TLR signalling exemplified by TLR4. Ligands are coloured blue, receptors are red, adaptor proteins purple, pro-inflammatory transcription factors green, and cellular outputs orange.

NLR signalling pathways can be broadly split into two: 1) upregulation of NFkB and activation of pro-inflammatory genes; 2) Inflammasome formation, caspase- 1 activation and secretion of IL-1 $\beta$ and IL-18 (Figure 4). The first pathway is utilised by the prototypical NLRC family members NOD1 and NOD2. These receptors bind to PAMPs derived from bacterial peptidoglycan (Table 1). The activated NLRs signal through the adapter Receptor Interacting Protein 2 (RIP2) to drive the release of NFKB into the nucleus by the same processes employed by TLRs. In a further demonstration of PRR cross-talk, Nod2 also activates the MAP kinase pathway using a different adapter, CARD9, to upregulate pro-inflammatory gene expression from AP1 dependent promoters. The inflammasome forming NLRs consist, to date, of NLRP1, NLRP3 and NLRC4. The NLRP family members recruit the protein ASC (apoptosis-associated speck-like protein containing a CARD) through homotyoic Pyrin:Pyrin interactions. ASC also possesses a CARD domain which recruits pro-caspase 1 to the inflammasome complex. Selfcleavage of pro-caspase 1 releases active caspase- 1 which subsequently cleaves pro-IL-1 $\beta$ and pro-IL-18 into their mature forms for secretion from the cell. Both IL-1 $\beta$ and IL-18 are proinflammatory and they play crucial roles in host defence against pathogens. IL- $1 \beta$ is responsible for the generation of systemic and local immune responses by causing fever, activating lymphocytes and recruiting them along with neutrophils to the site of infection. IL18 lacks the pyrogenic nature of IL- $1 \beta$ but is involved in induction of IFN- $\gamma$ production by T- 
cells and NK cells to drive T-helper cell type 1 (Th1) responses during adaptive immunity development. The NLRC4 inflammasome can activate caspase-1 in an ASC-dependent and independent manner. Pro-IL-1 $\beta$ and pro-IL-18 are both expressed in an NFKB dependent manner. Hence their cellular levels are increased by TLR and NLR activation. The inflammasome can then be activated in response to a diverse selection of ligands, increasing the levels of IL-1 $\beta$ and IL-18 and amplifying the initial response to many PAMPs.

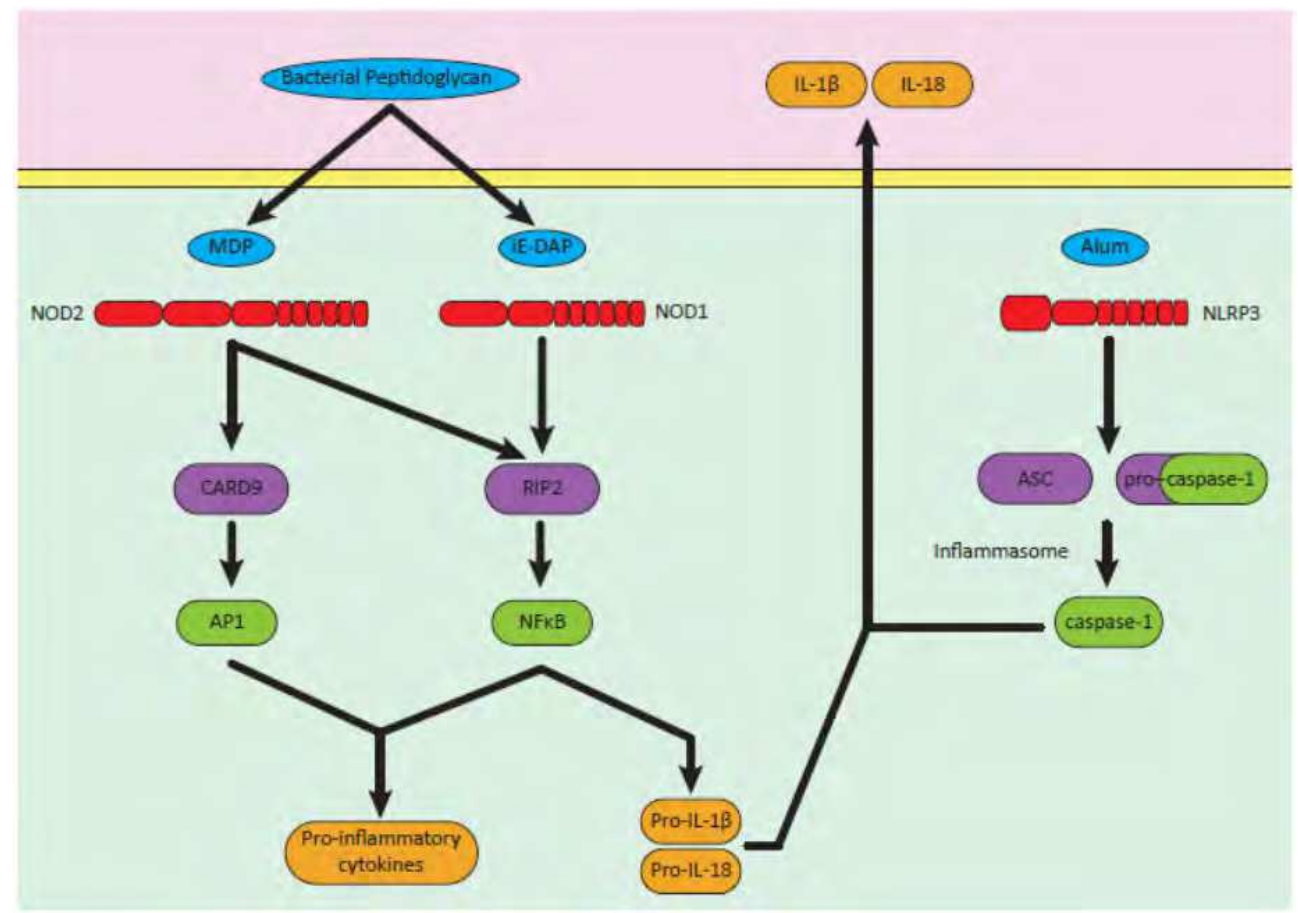

Fig. 4. Simplified representation of NLR family member signalling. Ligands are coloured blue, receptors are red, adaptor proteins purple, pro-inflammatory transcription factors green, and cellular outputs orange. Caspase- 1 activation ultimately results in cell death through the process of pyroptosis.

\subsection{Retinoic acid-inducible gene I-like receptors (RLRs)}

RLRs are a small family of PRRs that detect intracellular RNA. RLRs use CARDs to interact with downstream signalling components. Activation results in expression of type I IFNs and other pro-inflammatory cytokines. In conjunction with endosomal TLRs the RLRs provide a robust antiviral response. RIG-I recognises short uncapped dsRNA or ssRNA, whereas melanoma differentiation-associated 5 (MDA5) detects longer dsRNA such as poly (I:C). Both proteins signal by forming CARD:CARD interactions with MAVS (mitochondrial antiviral signalling protein; also known as IFN- $\beta$-promoter stimulator 1 (IPS-1)) which is localised on the exterior membrane of the mitochondria. This activates the IRF transcription factors, but again leads to co-stimulation of the MAP kinase pathway as seen in the signalling of the other PRRs. The RLRs are also involved in sensing and triggering a 
response to cytoplasmic DNAs which can be transcribed by RNA polymerase III into dsRNA.

\subsection{C-type lectin receptors (CLRs)}

CLRs are characterised by the presence of a C-type lectin domain. Over 1000 proteins in the human genome could be described as CLRs, however, only a few specifically modulate the innate immune response. CLRs are involved in diverse, often regulatory, roles within the immune system, such as antigen presentation and phagocytosis. Dectin- 1 and dectin-2 are the best characterised CLRs and signal through their immunoreceptor tyrosine-based activation motif (ITAM). The ITAM domain activates the spleen tyrosine kinase (Syk) which upregulates many of the pathways triggered by the other PRRs.

\subsection{Summary}

It is clear that although there is much diversity in the range of PAMPs that can be detected by PRRs, there is a common signalling strategy geared towards causing inflammation to both contain and then remove the infection. The mechanism of activation and signalling cascades involved in this process are highly complex and contain a significant level of overlap, redundancy and cross-talk. A more comprehensive discussion of these processes can be found in a variety of excellent review articles and the references they contain (Davis et al., 2011; Kawai and Akira, 2011; Loo and Gale, 2011; Osorio and Reis e Sousa, 2011).In the following sections we will see that although pathogens usually activate a number of PRRs, they have adapted their modes of attack so as to bypass the innate immune system, and hence be able to colonise the body.

\section{Pattern recognition receptor responses to pathogens}

The previous section highlighted the diversity of PRRs available to respond to PAMPs. To demonstrate the importance of PRRs in the response to infectious diseases we have chosen four major pathogens; the bacteria Salmonella spp., the virus Influenza A, the fungi Candida albicans, and the parasite Schistosoma mansoni. Here we describe the importance of PRRs in the recognition of these pathogens and induction of an innate immune response against them. As will become apparent the innate immune system has evolved so that multiple PRRs recognise different PAMPs from the same pathogen.

Pathogens, like higher organisms, also undergo evolutionary pressure to survive. In essence this can be viewed as a host-pathogen arms race. Successful pathogens are able to evade, limit, or manipulate detection by PRRs, and in some cases, utilise PRR signalling pathways for their own benefit. Pathogen survival can be augmented in a number of ways. These include targeting the recognition receptor, the signalling transduction event, and the key effector proteins of the innate immune system (Hajishengallis and Lambris, 2011). The innate immune system is a common target for immune evasion strategies for two primary reasons. Firstly, it is the initial host defence encountered by the pathogens upon infection. Secondly, the innate immune system is essential for the development of adaptive immunity. Consequently, by exploiting the innate immune system, pathogens can undermine the whole immune response of the host. Many different mechanisms are employed by pathogens to subvert immune signalling. These include: the use of immunomodulatory proteins; receptor antagonists; the 
induction of immunosuppression; activation of host immune inhibitory receptors; reduced expression, or alteration, of the PAMP; and manipulation of PRR crosstalk. In short successful pathogens are able to survive for longer in, and colonise, the host through maintenance of a careful balance between innate immune activation and suppression. In addition to identifying the key PRR-pathogen interactions we will provide examples highlighting the ability of these organisms to survive in the host and evade the innate immune response.

\subsection{PRRs and Salmonella}

\subsubsection{Salmonella is a Gram-negative bacterium that causes food-borne diseases}

Salmonella is a Gram-negative (Figure 5), rod-shaped, flagellated, bacterium which invades, and replicates and survives within, immune cells such as macrophages (Coburn et al., 2007). There are over 2500 serotypes of Salmonella enterica. S. enterica typhi and S. enterica typhimurium cause typhoid fever and enterocolitis respectively in humans. These diseases affect millions of people globally causing around 600,000 deaths annually, mostly in infants and immunocompromised patients.

Salmonella is transmitted via the faeco-oral route following ingestion of contaminated food, water or animal products, or close contact with an infected individual. Infection predominantly occurs in the epithelial lining of the intestine with intestinal epithelial cells (IECs) and macrophages being key cells for both bacterial uptake and immunity. IECs act not only as physical barriers to infection, but also contribute to the innate immune response following PRR activation. Bacterial uptake by macrophages (and dendritic cells) results in stimulation of a wide range of PRRs and can also serve as a route to systemic infection. The pathophysiology of Salmonella infections is strongly connected to the strong inflammatory response from the host. Interaction between host PRRs and Salmonella virulence factors influence the pathology, morbidity, and mortality at different stages of infection.

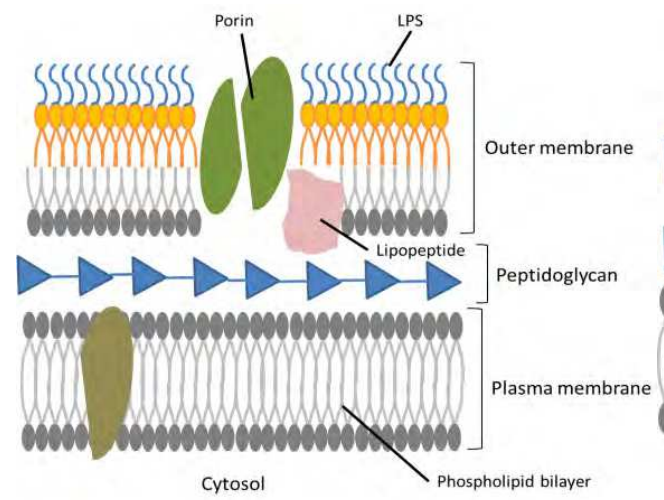

Gram-negative bacteria

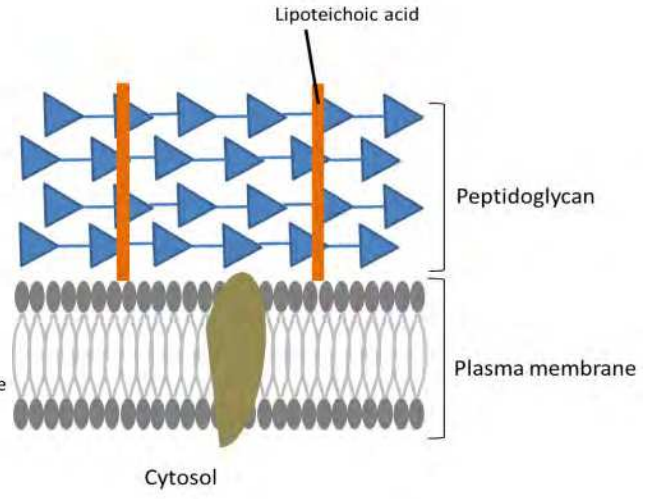

Gram-positive bacteria

Fig. 5. Schematic of the cell wall structure of Gram-negative (left-hand image) and Grampositive (right-hand image) bacteria highlighting the key components of each type of bacterial wall. Salmonella is a rod-shaped Gram-negative bacterium. Two key PAMPs associated with Salmonella are LPS and lipopeptide which activate TLR4 and TLR2 respectively. In addition, the surface of Salmonella possesses flagella, another potent immunostimulatory molecule. 


\subsubsection{Salmonella PAMPs and PRR activation}

Salmonella is recognised by a wide range of PRRs: TLR1, 2, 4, 5, 6, and 9; NLRC4; NLRP3; NOD1 and NOD2 (Hold et. al., 2011). Of these the key PRRs are TLR2, TLR4, TLR5 and NLRC4. Cell culture studies and mouse models have identified several Salmonella PAMPs responsible for PRR activation. Of particular importance are LPS, lipoproteins and flagellin (Figure 5). Flagellin provides an interesting example of a ligand that is able to activate two distinct receptors (TLR5 and NLRC4) leading to distinct immune responses (Franchi et al., 2006; Hayashi et al., 2001).

LPS and lipoproteins lead to classical activation of TLR4 and TLR2 signalling pathways respectively (Section 2.1). The importance of TLR2 signalling is influenced by the severity of infection, playing a key role at high multiplicities of infection. In contrast, the role of TLR4 appears essential at all levels of Salmonella infection (Spiller et al., 2008; Talbot et al., 2009). TLR4 activation by LPS requires several co-receptors such as: myeloid-differentiation 2 (MD2), cluster of differentiation 14 (CD14), and LPS-binding protein (LBP). The TLR4 signalling pathway is different from all other TLRs as its activation results in two separate signalling cascades: MyD88-dependent and MyD88-independent pathways which result in $\mathrm{NF}-\mathrm{KB}$ activation and IFN- $\beta$ and NFKB activation respectively (Figure 3 ). This activation is regulated temporally and spatially as it is been suggested that MyD88-dependent signalling occurs first on the plasma membrane, following which the receptor complex is endocytosed after which it will sequentially activate the MyD88-independent pathway. This is a classic example of the use of subcellular localisation of the receptor signalling complex to determine use of downstream adaptor proteins and the specifics of the signalling pathway activated. The importance of TLR4 in the response to Salmonella is highlighted by observations that mice possessing defects in TLR4 are incapable of mounting a normal immune response to Salmonella typhimurium infection (Talbot et al., 2009). It appears that the relative importance of different TLR and adaptor proteins in combating Salmonella is dependent upon the bacterial load. For example, the adaptor protein Mal only appears important at low multiplicities of infection (Kenny et al., 2009). This may well be a deliberate ploy on the part of the host to manage the severity of the response at a level which reflects the severity of infection.

TLR5 recognises monomeric flagellin. TLR5 is expressed on the basolateral surface, not the apical surface, of polarised epithelial cells so only responds to flagellin that has breached the epithelial barrier. This is likely to be a host strategy to stop inappropriate immune activation by flagellated commensal bacteria in the lumen (Gewirtz et al., 2001; Hayashi et al., 2001) . Once the bacterium has breached the epithelial barrier flagellin can then be detected by the baso-laterally located receptor. In addition, TLR5 is found expressed on the surface of immune cells such as macrophages and DCs.

The mechanism of flagellin recognition by TLR5 is interesting as only monomeric flagellin is recognised. This is because the TLR5 binding site is buried in the functional flagellar filament. This is one of the bacterium's ways of evading the host immune system. A single filamentous flagella consists of 11 protofilaments, each of which contains four globular domains (D0-D3) (Figure 6). The recognition site for TLR5 is contained in the N-terminal D1 domain. This region is essential for motility and is consequently highly conserved (Smith et al., 2003). Mutations within D1 have been shown to abolish recognition by TLR5, bacterial motility, and to disrupt protofilament assembly. In particular differences in amino acids 89- 
96 have been implicated in the ability of some species of bacteria to evade immune detection by TLR5 (Andersen-Nissen et al., 2005).

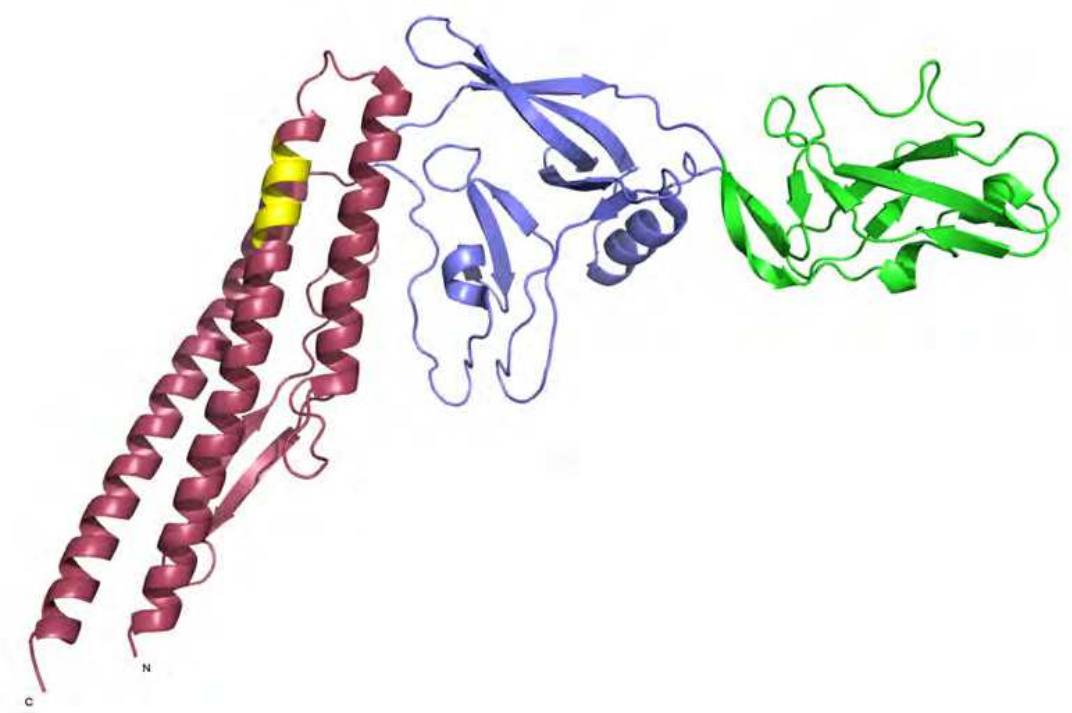

Fig. 6. Crystal structure of FliC flagellin from S. typhimurium (pdb entry 1io1). Domain colouring is: D1 - light purple; D2 - blue; D3 - green; the yellow region in D1 corresponds to amino acids 89-96.

Prior to detection by TLR5, flagella has to be depolymerised into its monomeric form. Direct contact between the bacterium and the host cell triggers the de novo synthesis and secretion of monomeric flagellin into the host cell via T3SS (Subramanian and Qadri, 2006). This process is triggered by the bacterium sensing lysophospholipids produced by the host cells upon infection and is important for initial inflammatory and innate immune responses by IECs. Following activation TLR5 forms a homodimer and initiates MyD88-dependent signalling cascades (Figure 3) as part of the innate immune response. One effect of this is to recruit neutrophils and macrophages to the site of infection.

Once Salmonella has breached the epithelia it can be phagocytosed by macrophages. The bacterium can reside and replicate inside a Salmonella-containing vesicle. Fragments of flagellin enter into the cytoplasm, probably via a type III secretion system, from where it stimulates the NLR family receptor NLRC4 (Franchi et al., 2006). NLRC4 is also activated by the protein PrgJ, a component of the type III secretion system expressed by the Salmonella pathogenicity island (SPI)-1 (Miao et al., 2010). The mechanisms of NLRC4 activation by bacteria such as Salmonella are complex and seem to follow some form of temporal cascade. The net effect is that activation of NLRC4 leads to inflammasome formation, caspase- 1 activation and secretion of IL-1 $\beta$ and IL-18. In addition, caspase- 1 activation via NLRC4 can trigger macrophage death through pyroptosis. Pyroptosis is a caspase- 1 dependent, programmed cell death event that has features of both apoptosis and necrosis. The process of pyroptosis results in the release of pro-inflammatory cellular contents and also serves to limit intracellular bacterial replication (Roy and Zamboni, 2006) (Figure 4). 


\subsubsection{Modulation of the innate immune response by Salmonella}

Salmonella is capable of infecting, surviving, and multiplying within macrophages. This requires multiple virulence proteins which are predominantly encoded on the SPI-2 pathogenicity island. These factors enable the bacterium to resist the oxidative burst and maturation of phagosomes and lysosomes. In fact the bacterium modifies these endocytic vacuoles and generates an environment conducive to bacterial replication - the salmonella containing vacuole (SCV) (Figure 7).

In addition to acting as a trigger of NLRC4 signalling (Section 3.1.2) the T3SS allows proteins to be injected into the cell cytoplasm that modulate cellular function. A major role of these proteins is to alter cytoskeletal function and permit bacterial entry. In addition, Salmonella also secretes an immunomodulatory protein SipB. The precise role and mechanism of action of SipB is unknown. However, the protein is capable of interacting with caspase- 1 and consequently altering the signalling pathways involved in caspase- 1 activation and downstream functionality (Hersh et al., 1999). Other bacteria also produce proteins that modulate immune signalling. For example, it has recently been shown that Escherichia coli and Brucella melitensis produce TIR domain containing virulence factors that inhibit TLR signalling by directly blocking MyD88 adaptor protein function through a homotypic TIRTIR interaction (Cirl et al., 2008).

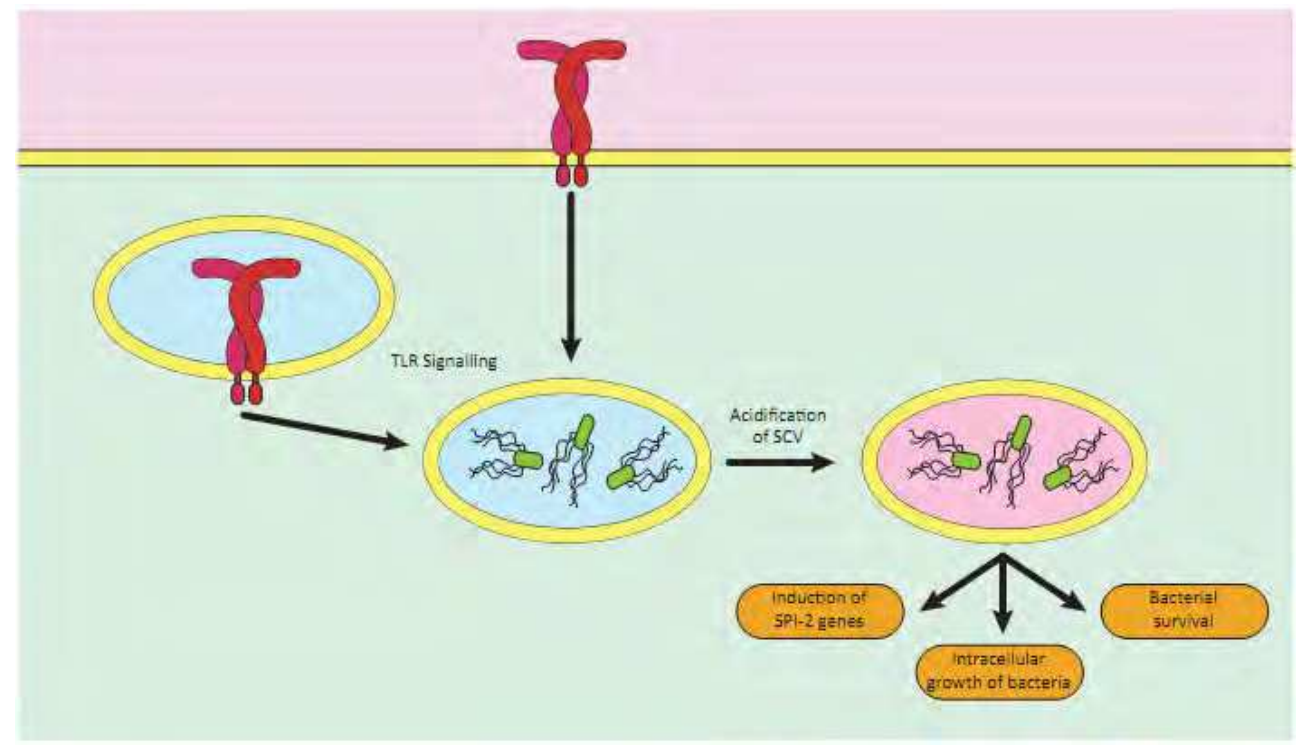

Fig. 7. Formation of a Salmonella containing vacuole (SCV) promotes Salmonella survival and virulence inside host macrophages. SCV formation appears to be dependent on functional TLR signalling and requires acidification to activate expression of the Salmonella prosurvival genes. 


\subsubsection{Altering PAMP properties as a way to evade immune detection}

Salmonella flagellin is a readily available and common target recognised by both extracellular, TLR5, and intracellular, NLRC4, PRRs. Although many PAMPs cannot be altered and changed in order to evade the immune response this is not the case for flagellin. Salmonella has been found to downregulate flagellin expression once it is inside macrophages, although how quickly this happens is unclear. A loss of flagellin expression will obviously reduce the potential for immune stimulation, but it has yet to be determined whether this occurs in a timeframe that will influence PRR activation. As TLR5 recognises monomeric flagellin mechanisms that increase flagellum stability and reduce the release of monomeric subunits will also reduce immune activation.

Some other flagellated bacteria have adopted a mechanism in which they express flagellin lacking the proinflammatory regions found in Salmonella FliC (Section 3.1.2). The classic example is Helicobacter Pylori. H.pylori express the flagellin proteins FlaA and FlaB. These subunits retain the ability to assemble motile flagella, but lack the TLR5 stimulatory regions. This was elegantly demonstrated by experiments in which the comparable sequence from H.pylori flagellin was substituted into Salmonella FliC. The mutant flagellin lost the ability to activate TLR5.

\subsection{PRRs and viral PAMPs}

Viruses are more abundant than bacteria; they are also very diverse genetically and evolve rapidly. Viruses are obligate parasites and can only survive and replicate inside host cells. They have different virulence components and simpler structural features in comparison to bacteria. The requirement on the host cell for replication makes viral nucleic acids the key viral PAMPs. Consequently, only a subset of PRRs can detect them. These include: NLRP3; NOD2; TLR3, 7, 8, and 9; and the RIG-I like receptors (Kanneganti, 2010; Perry et al., 2005) (Table 2).

\begin{tabular}{|l|l|l|l|}
\hline PAMP & PRR & Adapter & Cytokines \\
\hline Toll-like receptors (TLRs) & TLR3 & TRIF & IFN- $\beta$ / pro-inflammatory \\
\hline dsRNA & TLR7 & MyD88 & IFN- $\alpha$ / pro-inflammatory \\
\hline G/U rich ssRNA & TLR8 & MyD88 & IFN- $\alpha$ \\
\hline G/U rich ssRNA & IFN- $\alpha$ \\
\hline unmethylated CpG DNA motifs & TLR9 & MyD88 & \\
\hline Nod-like receptors (NLRs) & NOD2 & MAVS & Type I IFNs \\
\hline ssRNA & NLRP3 & ASC & IL-1 $\beta /$ IL-18 \\
\hline Viral RNA & RIG-I & MAVS/ASC/CARD9 & Type-I IFNs/ IL-1 $\beta$ \\
\hline Retinoic acid-inducible gene I-like receptors (RLRs) \\
\hline 5
\end{tabular}

Table 2. Natural viral PAMPs are recognised by PRRs and stimulate cytokine production. Viral nucleic acids generally differ from host nucleic acids through the presence of specific motifs and also their subcellular localisation. Viral nucleic acid (blue column) is recognised by specific PRRs (pink column) and this leads to receptor activation. The activated receptor recruits specific adaptor proteins and initiates signalling cascades. The net result of this is the secretion of cytokines. 
The host response to viral infection results in the production of anti-viral agents such as type I IFNs that interfere with viral replication and survival inside host cells, and proinflammatory cytokines such as IL-1 $\beta$ and IL-18. Type I IFNs are the key players in innate immune response against viral infection. We will expand the discussion on role of PRRs in viral infection by considering the example of influenza virus.

\subsubsection{Recognition of influenza by TLRs}

Influenza is a negative-sense ssRNA virus that causes pulmonary inflammation and chronic lung diseases. The symptoms vary from fever and inflammation to death, depending on the strain of the virus and the host response. Influenza enters host cells by endocytosis following recognition of sialic acid residues by the viral haemagglutinin protein. This is followed by $\mathrm{pH}$-dependent fusion of the viral and endosomal membranes allowing release of the viral core into the cytosol. The exact sequence of events that subsequently leads to recognition of influenza by PRRs is still largely unknown. Influenza PAMPs can be recognised by a variety of PRRs (Figure 8 and Table 2). However, the majority of immunopathology appears to result from TLR7, NLRP3 and RIG-I activation (Ichinohe, 2010). Plasmacytoid dendritic cells (pDCs) are the major cell-type responsible for mounting an immune response to influenza through the secretion of high amounts of type I IFNs and proinflammatory cytokines (Perry et al., 2005). Macrophages, nasal airway epithelial cells and monocytes have also been found to respond to influenza. Secreted type I IFNs interact with IFN receptors causing expression of type I IFN-stimulated genes with antiviral properties. For example, increased expression of RIG-I like receptors and proteins involved in the inhibition of viral transcription and trafficking. Type I IFNs also influence the development of adaptive immunity such as increasing expression of costimulatory molecules on macrophages and DCs, aiding maturation of DCs, and the activation of $\mathrm{T}$ and NK cells.

pDCs are professional antigen presenting cells. Following endocytic uptake of virus particles the virus is degraded and can contact endosomal PRRs. The presence of ssRNA in the endosome activates TLR7 (Lund et al., 2004) which signals in a MyD88-dependent manner to activate IRF3 and IRF7 transcription factors for the expression of type I IFNs. ssRNA recognition by TLR7 appears to require endosomal acidification before the receptor can form a homodimer and initiate the signalling pathways. TLR7 and MyD88 are both required for the type I IFN response to influenza ssRNA in pDCs. Studies have shown that mice deficient in either TLR7 or MyD88 are unable to respond to influenza virus (Diebold et al., 2004; Lund et al., 2004). TLR7 is also expressed in other cell types such as macrophages and conventional DCs, however TLR7 activation in these cell types only result in expression of proinflammatory cytokines. This difference is due to different signalling adaptor proteins present in these different cell types. Human naïve B cells and effector memory $\mathrm{CD}^{+} \mathrm{T}$ cells also express and signal through TLR7 activation (Wang et al., 2006).

The TLR7 response to influenza ssRNA is independent of viral replication. In contrast, there is evidence that dsRNA can induce type I IFN production via a TLR7- and MyD88independent pathway that requires viral replication (Guillot et al., 2005). Even though influenza is a ssRNA virus, dsRNA molecules are synthesised during the replicative stage of the virus. Certainly TLR3 recognises endosomal dsRNA from influenza virus (Guillot et al., 2005). The downstream signalling proceeds not through MyD88, but the adaptor TRIF to 
activate IRF3 and late-phase NF-кB. The importance of dsRNA recognition in influenza infection is supported by the observation that the influenza protein NS1, which sequesters viral dsRNA, inhibits type I IFN induction upon viral infection (Lu et al., 1995).

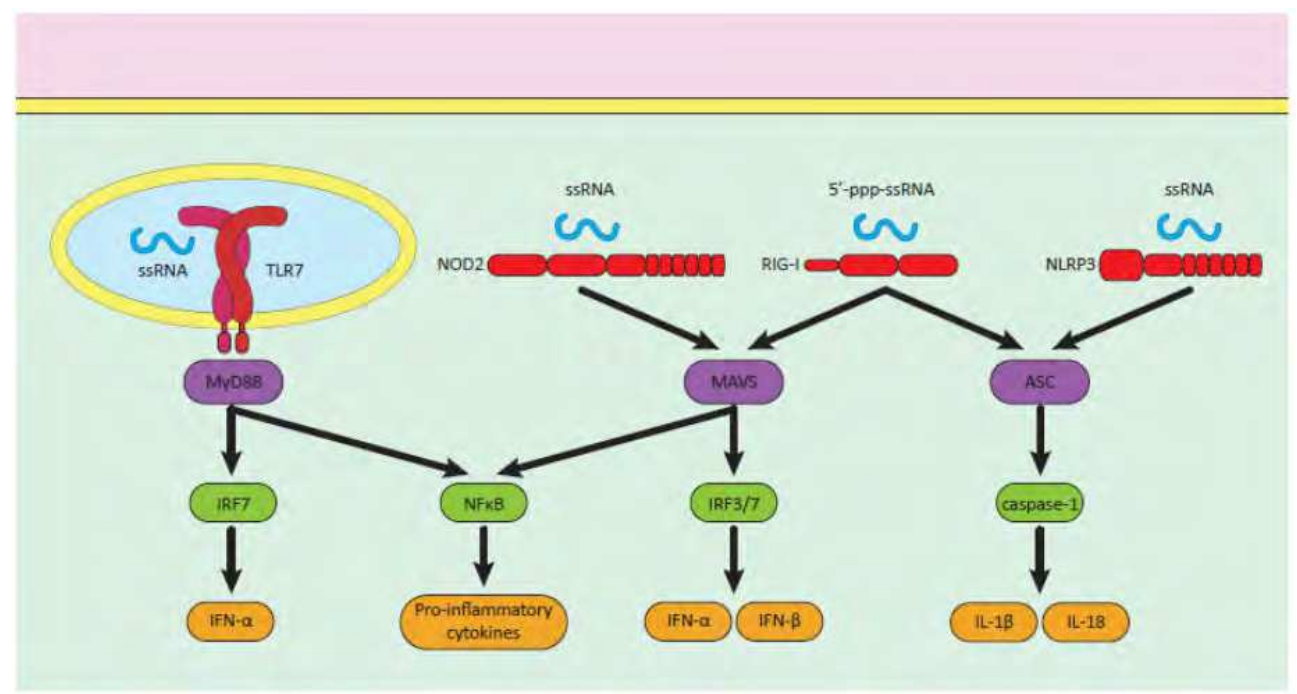

Fig. 8. Cytoplasmic and endosomal PRRs that recognise PAMPs from ssRNA virus such as influenza. Different PRRs can activate different pathways, and some pathways are shared by several PRRs.

\subsubsection{Recognition of influenza by NLR and RLRs}

Influenza can be recognised by the NLRP3 inflammasome, RIG-I and potentially NOD2 (Kanneganti, 2010). Activation of these signalling pathways results in the activation of caspase-1, IL-1 $\beta$ and IL-18; the production of inflammatory cytokines; and the secretion of type I IFNs via MAVS engagement. Each of these receptors is located in the cytoplasm and hence responds to the presence of RNA, 5'-phosphate ssRNA, or ssRNA within the cytoplasm. This contrasts to TLR7 which responds to ssRNA in the endosomal compartment. In the case of NLRP3 it is unknown whether viral RNA is a directly activating ligand for the receptor. Indeed it is more likely in this instance that the presence of viral RNA in the cytoplasm leads to a homeostatic disruption that in turn activates NLRP3, stimulates inflammasome formation, and initiates a protective innate immune response.

NOD2 and NLRP3 elicit different immune responses to viral RNA (Fig. 6). NLRP3 stimulation leads to activation of caspase 1 and subsequent processing of the pro forms of IL-1 $\beta$ and IL-18 via inflammasome formation. Both IL-1 $\beta$ and IL-18 are important in the clearance of influenza infection as mice which lack the IL-1 receptor and consequently can't respond to these cytokines show impaired viral clearance and increased mortality (Schmitz et al., 2005). Unlike NLRP3, NOD2 does not form an inflammasome. The primary role of NOD2 seems to be the detection of the bacterial peptidoglycan fragment muramyl dipeptide and signalling through NF-KB regulated pathways. However, more recently NOD2 has been 
reported to respond to viral ssRNA (Sabbah et al., 2009). Viral ssRNA results in activation of an alternative MAVS-dependent NOD2 signalling cascade in which NOD2 relocalises to the mitochondria and ultimately activates IRF3. This leads to the induction of an antiviral type I IFN response. A loss of NOD2 led to an increase in the susceptibility of mice to influenza infection, a reduction in IRF3 phosphorylation and a diminishment of the type I IFN response (Sabbah et al., 2009).

RIG-I can also signal in a MAVS-dependent manner to generate a type I IFN response (Kanneganti, 2010). Interestingly RIG-I has also been reported to stimulate the production of pro-IL-1 $\beta$ production in a MAVS-CARD9-NFkB dependent manner; as well as inducing proIL-1 $\beta$ processing and secretion in an ASC-dependent inflammasome manner (Kanneganti, 2010; Pichlmair et al., 2006). This therefore provides a single receptor example of cross-talk along multiple signalling pathways in order to maximise the anti-viral and inflammatory reponse.

\subsubsection{Subversion of the innate immune response by influenza}

Viruses have long been known to utilise multiple strategies to evade the immune response, persist in the host, or allow reinfection. In the case of influenza virus the influence of antigenic drift in the surface haemaglutinin and neurominidase proteins allows evasion of the adaptive antibody response thereby limiting the protective effects of vaccination and facilitating influenza epidemics. Antigenic shift is a more extreme, and less common, evasion strategy in which viruses from different species undergo recombination to create a new potentially pandemic viral strain. Commonly recombination occurs between human and avian viral strains.

Influenza virus also employs various strategies to disrupt the innate immune response; either by interfering with PRR mediated detection of viral components, or through modulation of signalling cascades. The viral non-structural protein (NS)1 is a good example of a protein that targets a specific signalling pathways to avoid detection. NS1 has the potential to inhibit the activation of caspase 1 and the production of type I IFNs. Interestingly there does appear to be strain-dependent variation in activity of NS1 in this respect. The N-terminal region of NS1 appears to be important for the inhibition of inflammasome-dependent immune responses; specifically through inhibition of caspase-1 activation, and hence IL-1 $\beta$ and IL-18 production (Kanneganti, 2010). NS1 has also been reported to inhibit RIG-I-MAVS-dependent antiviral signalling (Bowie and Unterholzner, 2008). Pichlmair and colleagues, reported that NS1 binds directly to RIG-I and inhibits its activation, thereby reducing type I IFN production (Pichlmair et al., 2006). However, the exact molecular mechanism of how NS1 inhibits RIG-I signalling is not clear. This interaction may directly antagonise RIG-I function, or it could involve other proteins that are required for proper signalling. For example, NS1 also sequesters dsRNA so that they cannot be detected by dsRNA-recognising PRRs such as TLR3 and PKR. More recently, Gack and co-workers showed that NS1 inhibits RIG-I signalling by inhibiting activation of ubiquitin ligase tripartite motif (TRIM)25; a crucial component of the RIG-I signalling cascade required for activation of RIG-I through ubiquitination within the CARD domain (Gack et al., 2007). 


\subsection{PRRs and Candida albicans}

\subsubsection{Candida albicans}

The yeast Candida albicans is a common human commensal that colonises mucocutaneous surfaces of the oral cavity, gastrointestinal tract and vagina. Candida albicans is often a benign member of the mucosal flora. However, under specific host conditions, such as a change in immune status or commensal species, Candida albicans can proliferate in a saprophytic state and become an opportunistic pathogen. This usually results in mucosal diseases, but on occasion the infection may become systemic and life-threatening. A specific example is nosocomial candidiasis in hospital patients with a compromised immune system. Candida albicans can differentiate in yeast, pseudohyphal and hyphal forms. The hyphal form is often linked to disease as it facilitates infection of epithelial cells and tissue damage. In addition to hyphae Candida species enhance their pathogenicity through biofilm formation and secretion of proteases and toxins. The balance between the commensal and pathogenic state is what makes Candida albicans a useful study case for looking at the interplay between host PRRs and PAMPs, and the dynamics that lead to protection/tolerance or infection.

\subsubsection{Induction of an immune response by Candida albicans}

It is hypothesised that the initial interaction between the host immune cell and specific C.albicans PAMPs is critical for deciding whether the immune response is protective or invasive. Studies suggest that a prolonged anti-inflammatory response against C. albicans, including increased production of IL-10, IL-4 and differentiation of regulatory/suppressive $T$ cell subsets facilitates invasion and the initial development of disease. In the absence of IL10 there is a switch to a protective response by the innate immune system. This includes stimulation of inflammatory cytokines such as IL-12, TNFa, and IL-6; and progression towards a Th17 response leading to IL-17 and IL-22 production to clear the infection. Further evidence for the role of IL-10 as a modulator of yeast infection is suggested by animal models. IL-10 deficient mice are protected against the toxic effects of C.albicans infection and show a decline in inflammatory markers associated with yeast infections. In contrast, inclusion of IL-4 and IL-10 in a gastrointestinal model of yeast infection facilitates invasion rather than protection.

Interaction studies between host immune cells and Candida albicans have identified various PRRs that recognise yeast derived PAMPs, and facilitate protective responses via the proinflammatory pathway. Most yeast and fungal PAMPs have been identified in the cell wall, a complex matrix consisting of glucans, mannans, mannoproteins and glycolipids. Several receptors including TLR4 and the C-type lectins Dectin-2, Mincle and the macrophage mannose receptor bind to mannans of Candida species. Dectin-1, lactosylceramide complement receptor- 3 and scavenger receptors recognise $\beta$-glucans of Candida.

\subsubsection{TLR sensing of Candida albicans}

TLRs are involved in the immune response against $C$. albicans as well as other fungal agents, particularly TLR2 and to a lesser extent TLR4. Both pathogenic and commensal forms of $C$. albicans are involved in TLR-dependent signalling, in addition to other commensal yeasts such as Saccromyces cerevisiae. TLR2 recognises phospholipomannan components of the 
fungal cell wall. TLR4 is activated by a-mannans on C. albicans and initiates the production of IL-12 by host immune cells and a subsequent protective Th1 response. TLR4 polymorphisms Asp299Gly/Thr399Ile are associated with an increased susceptibility to C. albicans infection in the bloodstream, with an increase in IL-10 levels being the contributing factor (Van der Graaf et al., 2006). In addition to a pro-inflammatory response, TLR2 activation may also lead to the production of IL-10 and generation of T regulatory cells and a non-protective Th2 response. This is supported by data on TLR2 KO mice which are more resistant to Candida infection. The TLR2 polymorphism R753G gives rise to patients with enhanced Candida sepsis, suggesting that TLR2 is an important PRR for regulation of Candida infections (Woehrle et al., 2008). Indeed, TLR2 signalling complexes may modulate the Th1/Th2 (IL-10) balance in response to fungi by switching between pro- and antiinflammatory responses. TLR signalling occurs in crosstalk with the carbohydrate specific PRRs, the C-type and S-type lectins, to regulate C. albicans infection.

\subsubsection{CLR recognition of Candida albicans}

CLRs such as Dectin-1, Dectin-2, Mincle, DC-SIGN (SIGNR1, mouse homolog) and mannose receptor (MR) are key PRRs in the immune response against fungi. The interaction of CLRs with PAMPs is mediated via carbohydrate recognition domains (CRD), which facilitate internalisation, degradation and antigenic presentation. CLRs signal via the Syk pathway to initiate an inflammatory response using an ITAM motif in their cytoplasmic tail. Some CLRs such as Dectin-2, lack an ITAM motif and instead utilise adaptor molecules such as the Fc receptor $\gamma$ chain and the DNAX-activating protein (DAP12) which have an ITAM motif for signalling.

Dectin-1, which is expressed in neutrophils, macrophages and DCs, is a specific receptor for $\beta-1,3$ glucans, located in the cell walls of fungi, such as Saccharomyces cerevisiae (zymosan) and Candida albicans. Dectin-1 signalling pathways are not completely understood. It is proposed that Dectin-1 mediated responses may require crosstalk with TLRs, NLRs, tetraspanins and the DC-SIGN receptor. Dectin- 1 binds and internalises $\beta$-glucans, and mediates its own signalling pathway which includes the production of reactive oxygen species (ROS) also termed an oxidative burst, activation of $N F K \beta$ and secretion of proinflammatory cytokines. Following ligand recognition via the CRD, Dectin-1 is phosphorylated by the tyrosine kinase Src, which recruits Syk to the ITAM motif to activate MAPKs and NFAT. This Syk interaction also mediates formation of the CARD9-Bcl10-Malt1 complex, which stimulates NFK $\beta$ and the secretion of proinflammatory cytokines. This ultimately leads to a Th17 T cell response. Th17 cells are particularly important for antimicrobial immunity at the epithelial and mucosal barriers and produce cytokines such as IL17 and IL-22 which stimulate anti-microbial proteins. A lack of Th17 cells leaves the host susceptible to invasion by opportunistic pathogens such as C.albicans.

A recent study suggests that Malt- 1 activation of the NFk $\beta$ subunit C-Rel is important for induction of Th17 enhancing cytokines IL1 $\beta$ and IL23. It was also evident that Dectin-1 activates all components of the NFK $\beta$ complex, whereas Dectin-2 specifically activates C-Rel (Gringhuis et al., 2011). Recent studies suggest that Dectin-1 NFK $\beta$ activation is induced by zymosan, whereas hyphal forms of C.albicans activate NFK $\beta$ via Dectin-2. Hyphae stimulation in C.albicans also facilitates association of CARD-9 with Bcl10, in a Dectin-2 specific manner (Bi et al., 2010). This is a good example of how different PRRs of the host 
have distinguished between pathogenic (hyphae producing) and commensal forms of Candida albicans.

The ability of Dectin-1 to activate Th17 responses assists in the prevention of fungal infection. Loss of Dectin-1 in KO mice and Dectin-1 polymorphisms in humans makes the host more susceptible to infection (Ferwerda et al., 2009b). In C.albicans infection, $\beta$-glucans may also stimulate Th17 and regulatory T cell responses via a Dectin-1/TLR2 crosstalk, in addition to the Dectin-1 specific pro-inflammatory cytokine response (Gantner et al., 2003; LeibundGut-Landmann et al., 2007). This Dectin-1/TLR2 response also involves the production of prostaglandin E2, which upregulates Th17 dependent cytokines IL-6 and IL23 (Smeekens et al., 2010). Dectin-1 also synergises with TLR2 and TLR4 for the production of TNFa and there is evidence to suggest that Dectin-1 and TLR2/6 pathways crosstalk to enhance responses by each receptor. Furthermore, Dectin- 1 activation also upregulates proIL-1 $\beta$ for subsequent activation by the NLRP3 inflammasome (Cheng et al., 2011).

Dectin-1 also associates with Galectin-3, an S-type lectin that binds to $\beta-1,2$ mannosides present in the cell wall of Candida albicans, an interaction that is required for proinflammatory responses in fungi. The Dectin1-Galectin-3 complex modulates TNFa levels, whereby a decrease in galectin-3 corresponds to a decrease in TNFa. Mutant C.albicans expressing more $\beta$-glucan on the cell wall surface had reduced galectin- 3 binding and a reduction in the protective pro-inflammatory response. Hence, association between Galectin-3 and Dectin-1 can modulate the proinflammatory response to help distinguish between pathogenic and nonpathogenic fungi (Esteban et al., 2011).

Dectin-2 recognises several fungal species including C.albicans and interacts with PAMPs of high mannose content, specifically a-linked mannans and zymosan. Dectin-2 also activates NFk $\beta$ and a Th17 response via the Syk-CARD9 pathway, as well as MAPKs via a CARD9 independent pathway. However unlike Dectin-1, it couples to Syk indirectly utilising the ITAM motif of the FC receptor. A recent study has suggested that it is Dectin-2, rather than Dectin-1, that is more involved in the Th17 response to C.albicans and their hyphae, with IL$1 \beta$ and IL-23 induction being Dectin-2 dependent. DCs from Dectin-2 KO mice show a limited cytokine response to a-mannans (Saijo et al., 2010).

Macrophage inducible C-type lectin (Mincle) binds to yeast cell wall components, specifically a-linked mannans, and like Dectin-2 also uses the Fc receptor to signal in a SykCARD9 dependent matter. Mincle has also been linked to TLR2 responses. DC-SIGN and its mouse homolog (SIGNR1) recognises complex mannoside structures on the surface of yeast facilitating internalisation. SIGNR1 specifically binds zymosan as well as live and heat killed C.albicans. SIGNR1 has also been reported to modulate TLR4 dependent signalling and to itself induce a proinflammatory response against C.albicans (Takahara et al., 2011). SIGNR1 may also interact with Dectin-1 to enhance Syk dependent pathways.

There is recent evidence to suggest that the interaction of candida albicans with glucan (Dectin-1) and mannan (Dectin-2) specific receptors, works in combination with the adaptor protein MyD88 to activate phospholipase $\mathrm{A}_{2}$ and the production of eicosanoids, which are important modulators of inflammation (Suram et al., 2010). 


\subsection{PRRs and Schistosoma mansoni}

\subsubsection{Schistosoma infection and immunity}

The parasitic worm Schistosoma represents the fourth most prevalent helminth infection worldwide affecting 200 million people. In schistosomiasis, the human parasitic disease caused by helminths, the parasite takes on various forms during its lifecycle, which presents a variety of PAMPs to the host and contribute to the developing host immune response. The lifecycle of S.mansoni in mammalian hosts begins when the larval stage (cercariae) is released from an intermediate host (snail; genus Biomphalaria) and then infects host cells, where it transforms into adult worms. Hosts infected with these parasites accumulate hundreds of intravascular worms which secrete various antigenic molecules into the blood which continuously trigger the host inflammatory response and facilitate prolonged colonisation. During S.mansoni infection, the adult worms can survive for many years, where they evade the host immune response, by establishing a balance between host activation and immune suppression. The adult worms also produce hundreds of eggs daily over a life period of 5 to 30 years. A Th2 anti-inflammatory response is triggered by the presence of eggs as well as soluble antigens secreted by the eggs, whereas a Th1 inflammatory specific response is more likely when adult worms interact with host immune cells.

\subsubsection{Recognition of Schistosoma mansoni by PRRs}

Schistosoma mansoni produces various glycoconjugates (glycoproteins and glycolipids) that interact with PRRs of the innate immune system and potentially modulate the function of host immune cells (Figure 9). The mouse model of schistosomiasis identified that Schistosoma glycoconjugates containing Lacto-N-fucopentaose III (LNFPIII) induce a Th2 response in collaboration with TLR4. Also the schistosomal tegument has been reported to activate inflammatory responses in DCs via a TLR4/MyD88 pathway. Schistosomiasis is also associated with high levels of endotoxemia and elevated levels of high mobility group 1 (HMGB1) protein, which is a ligand for TLR2, TLR4 and the RAGE receptor.

Lysophosphatidylserine (Lyso-PS) and dsRNA from the eggs of S.mansoni also activate TLR2 and TLR3 inflammation pathways respectively. Soluble schistosomal egg antigens (SEA) inhibit TNFa and IL-6 secretion originating from TLR signalling pathways. This TLR suppression occurs at the same time as NLRP3 activation and IL-1 $\beta$ production (Ritter et al., 2010). SEA binds to the Dectin-2/FcRY complex and signals via the Syk pathway to induce ROS and potassium efflux, known effectors of the inflammasome NLRP3. S.mansoni infection of mice without inflammasome components ASC and NLRP3 failed to induce an inflammatory response indicated by the lowered IL-1 $\beta$ levels and the downregulation of $T$ cell responses.

CLRs also recognise glycans associated with schistosomes using one or more CRD (Figure 9). The CLRs DC-SIGN, macrophage galactose type lectin (MGL) and MR on human DCs, bind specific glycans on the SEA of S.mansoni. This binding facilitates internalisation of the SEA and promotion of a Th2 response. DC-SIGN can also bind to the larval form of S.mansoni via glycolipids. Human DCs primed with adult worm glycolipids switch towards a Th1 immune response and induce an inflammatory cytokine cascade. DC-SIGN binds to fucose components of the glycolipid of the worm and helps activate TLR4 signalling and 
inflammation (van Stijn et al., 2010). It is proposed that DC-SIGN recruits glycolipids and then presents them to TLR4, via a TLR4-DC-SIGN complex in lipid rafts.

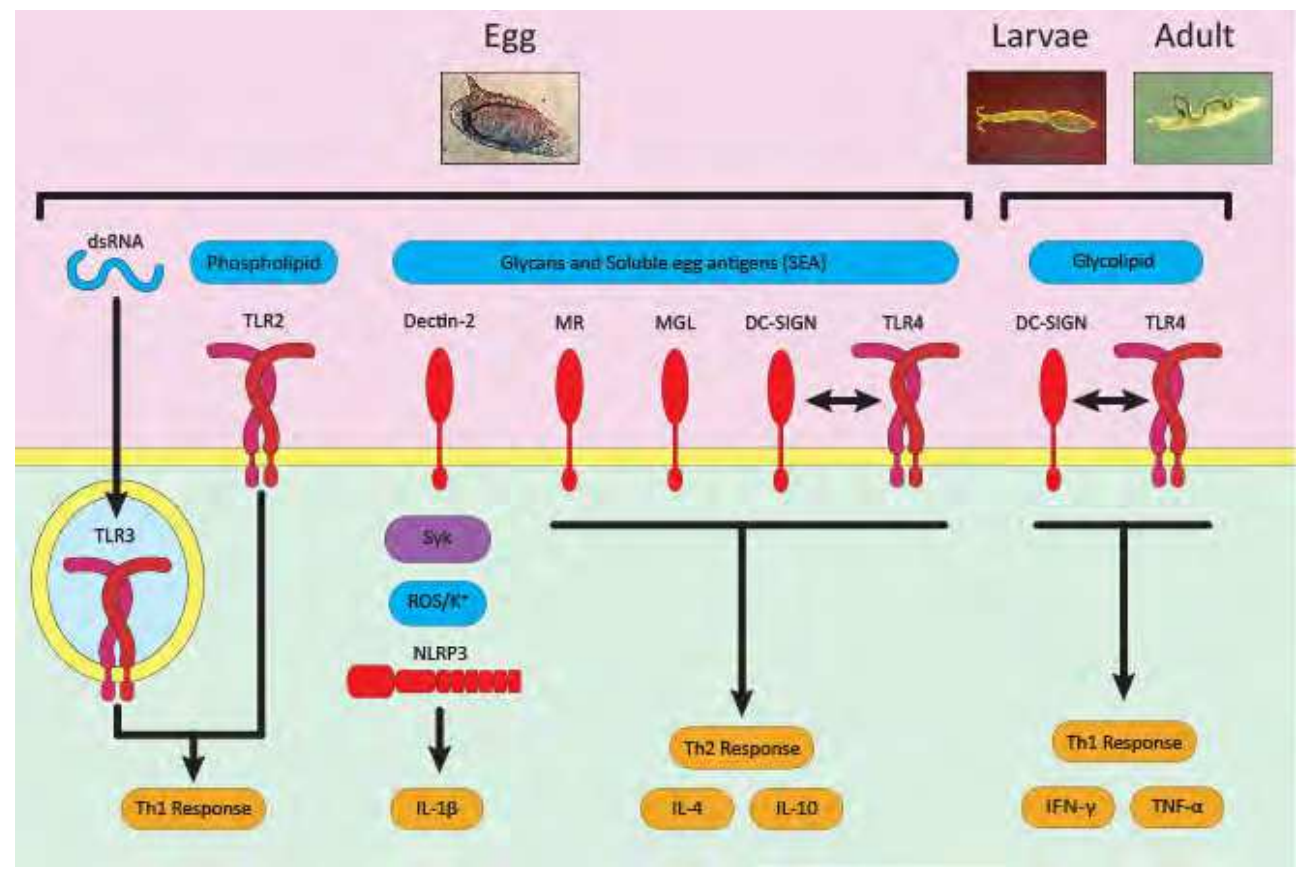

Fig. 9. PRRs involved in the immune response against schistosomal PAMPs at different stages of the schistosomes lifecycle. The activation of a Th1 (inflammatory) and a Th2 (humoral) response demonstrates the ability of the parasite to regulate infection. Pictures of schistosoma egg, larvae and adult reproduced with the permission of David Dunne.

\section{The role of PRRs in immunisation against infectious disease}

Immunisation is a crucial and highly successful defence against many infectious diseases including: measles, tetanus, diphtheria, rubella, polio and tuberculosis. Over recent years it has become apparent that PRRs play a major and crucial role in the processes of immune priming and polarization. These new findings have been applied to vaccine research in two major areas: firstly to determine the molecular mechanism of action of known vaccines and adjuvants; and secondly new adjuvant components have been developed that target specific PRRs in a quest towards rationally designed 'customised' vaccines.

\subsection{The role of PRRs in current vaccines}

PRRs are important for the priming of adaptive immune responses (Section 3.3). It is not surprising that many current vaccines also function, at least in part, by activating PRRs. Studies with knock-out mice have shown that PRRs are critical for the optimal function of several known vaccines. However, PRRs are not necessary for all vaccines to induce protective immune responses, and several vaccines instead trigger alternative pro- 
inflammatory pathways that appear to primarily recognize tissue or cellular injury rather than PAMPs.

Although much is known about the PAMP content of many vaccines, the role of PRRs in triggering protective immunity is more challenging to determine for three reasons. Firstly, individual PAMPs can be efficiently identified by measuring innate activation of cells such as DC in vitro whereas induction of immunity can only be measured in vivo with corresponding increase in cost and difficulty of interpretation. Secondly, the presence of multiple PAMPs and corresponding PRRs that coordinate host responses means that study of immunity in single knockouts often leads to partial phenotypes. Thirdly, many important human vaccines- particularly live attenuated vaccines - show host-species specific activity, preventing the direct study in PRR knockout mice.

\subsubsection{Evidence of PAMP content and PRR activation by traditional vaccines}

Traditionally, vaccines belong to one of three types: live attenuated vaccines, such as the eponymous vaccinia, are infectious agents in their own right, and prime protective immunity but lack pathogenicity; inactivated vaccines contain whole infectious agents that have been inactivated - for example by heat or chemical sterilization - to prevent infection, but still provoke protective immunity without replication; subunit vaccines contain a purified protective antigen combined with an adjuvant i.e. a formulation that promotes an immune response against the subunit. Of these three types, it is clear that both live attenuated and inactivated vaccines closely resemble pathogens - and therefore contain many PAMPs and trigger multiple PRRs. Subunit vaccines, in contrast, only contain PAMPs if they are copurified with the antigenic subunit, if the antigenic subunit is itself PRR agonistic, or if the adjuvant activates PRRs (see 4.1.2, 4.1.3 and 4.1.4).

\subsubsection{PRR activation by live attenuated vaccines}

Our oldest vaccines are live attenuated pathogens. Yet they are also the most poorly understood due to the complex interplay between parasite and host. Live attenuated vaccines contain a plethora of PAMPs. For example, live attenuated bacterial vaccines such as Mycobacterium tuberculosis BCG and live oral Typhoid Fever vaccine are rich in well known PAMPs such as bacterial DNA, lipopolysaccharides and lipopeptides. Two major challenges in characterizing PRR triggering by live attenuated viral vaccines are the fact that PAMP content and location changes during the infectious cycle (e.g. production of different types of nucleic acids, viral entry into host cells); and the presence of PRR inhibitors can obscure signaling.

Vaccinia is one of the best studied live attenuated vaccines. The importance of PRRs in vaccinia virus sensing became clear very early on with the discovery that its genome encodes inhibitors of IL-1 and TLR signaling (Bowie et al., 2000). Host responses to vaccinia are complex. Pathways act separately and synergistically, and there are diverse responses from different cell types. Studies of viral-induced cytokine production from cells from knockout mice have started to unpick this complexity and demonstrated that vaccinia can trigger multiple PRR including TLR2/TLR6, (Delaloye et al., 2009; Zhu et al., 2007), the RLR MDA-5 (Delaloye et al., 2009), and TLR8 (Martinez et al., 2010). The inflammasome component NALP3 is also required for maximal cytokine responses (Delaloye et al., 2009). 
Although the rich PAMP content of live attenuated vaccines has been characterised, understanding fully how PRR triggering induces protective adaptive immunity still remains a challenge. For example, MyD88-/- mice show reduced protection from experimental tuberculosis after vaccination with BCG (Fremond et al., 2004). And studies of the mouse Typhoid model Salmonella typhimurium suggest that TLRs and MyD88 are required in a range of cell types for optimal function of oral live attenuated bacterial vaccines (Dougan et al., 2011). Likewise, although the precise PRR requirement for protective immunity to vaccinia cannot be determined due to the lack of appropriate (or safe) models, TLR2 and MyD88 knockout mice do show partial reduction in MVA-specific T cell responses and CD8+ T cell IFN $\gamma$ secretion (Zhu et al., 2007).

\subsubsection{PRR activation by inactivated vaccines}

Inactivated vaccines are generally simpler to study because they have a fixed content, do not undergo metabolic changes, and in general do not subvert host signaling. Furthermore, a great deal is known about the PAMP content of inactivated organisms since killed microbes have been of great use for decades as a source of 'natural' PAMPs in the identification and study of PRRs.

Recently, a major pathway for viral nucleic acid recognition - viral ssRNA triggering TLR7 was initially identified by study of IFN $\alpha$ production by pDC in response to heat-inactivated influenza virus (Diebold et al., 2004) (Section 3.2.1). Indeed, in the absence of TLR7 whole inactivated influenza virus vaccine loses immunogenicity (Geeraedts et al., 2008). This study highlighted in particular the importance of TLR7 in driving a particular class of immune response - specifically Th1 immunity - reinforcing the notion that PRR triggering controls immune response polarization as well as magnitude. In contrast, split virus or subunit vaccines, which are less potent but considered safer, showed little TLR7 dependence presumably as a result of loss of the packaged viral ssRNA that potently stimulates TLR7 during production (Geeraedts et al., 2008). Of course these studies into the PRR requirements of vaccines have been performed in mouse models and to date the importance of PRR signaling for protective immunity in humans has yet to be determined, and may show different patterns.

Evidence for the need for TLR signaling for the activity of inactivated bacterial vaccines comes from the whole cell Pertussis vaccine. Although this vaccine contains a wide range of bacterial PAMPs, TLR4 and TRIF but not TLR2 knockout mice showed reduced immunity. This suggests that LPS is more critical than lipopeptides for induction of adaptive responses in this instance (Fransen et al., 2010).

\subsubsection{PRR triggering by subunit vaccines}

At first sight, subunit vaccines should not contain PAMPs because the critical microbial 'subunit' should be an antigen, i.e. target for adaptive - not innate - immune responses. But although simpler and purer than whole inactivated vaccines, many current human subunit vaccines still contain microbe-derived PAMPs either co-purified with the antigen or in some cases the antigenic subunit is itself a PRR agonist. Thus, meningitis vaccines based on purified outer membrane vesicle preparations of Neisseria meningitides contain both LPS and TLR2-stimulatory lipopeptides, and require TLR4 and TRIF signaling for optimal 
immune priming (Fransen et al., 2010). As well as PAMPs co-purified with antigenic subunits, many non-protein protective antigens such as polysaccharides or lipoproteins can directly trigger PRR. For example, the outer-surface lipoprotein (OspA) subunit of Borrelia burgdorferi activates TLR2 and has been used as an effective human vaccine against Lyme disease. This particular antigenic PAMP not only shows reduced immunogenicity in TLR2 knockout mice, but human hyporesponsiveness to the vaccine is associated with low TLR1 expression (Alexopoulou et al., 2002).

\subsubsection{PAMP-free triggering of PRRs by adjuvants}

The sections above have introduced the importance of TLRs in the development of immunity following vaccination. It is now becoming apparent that other PRRs, and stimulation of alternative proinflammatory pathways, can be important for vaccine functionality. There is evidence that some vaccine adjuvants can induce long-lasting and protective immune responses independently of TLR-based signalling pathways (Gavin et al., 2006; Nemazee et al., 2006). Furthermore, the majority of recently developed human adjuvant formulations, such as emulsions (Reed et al., 2009), do not obviously contain microbial components and are therefore unlikely to contain PAMPs. The NLRP3 inflammasome has been shown to be activated by the common adjuvant alum, however it is currently debatable as to whether this activation is required for the adjuvant activity of alum (De Gregorio et al., 2009; Franchi and Nunez, 2008; Li et al., 2008; McKee et al., 2009). Several other PAMP-free adjuvant formulations, including oil emulsions, saponins and microparticles made from biodegradeable polymer poly(lactic-co-glycolic acid) also trigger NALP3-dependent immune activation (Williams et al., 2010). In addition, the Syk/CARD9 pathway is activated by the potent adjuvant additive, trehalose 6,6-dibehenate, a synthetic mimic of a mycobacterial cell wall component (Werninghaus et al., 2009).

\subsection{Implications of PRR research findings for the development of new vaccines}

There is still a large demand for better vaccines in order to reduce costs, improve potency and efficacy, to tackle diseases which currently lack a vaccine (e.g. Human Immunodeficiency Virus) and to respond quickly to new challenges from infectious diseases (e.g. pandemic influenza). Understanding how PRRs contribute to protective immunity and how this can be modulated are key if vaccines are to improve.

One area of particular importance to vaccine development is the need to rationally design new adjuvants with greater potency than current ones such as alum (Mbow et al., 2010; Reed et al., 2009; Leroux-Roels, 2010). Selective triggering of different PRRs and DC subsets are thought to be major points of control of immune polarization (Iwasaki and Medzhitov, 2004; Kapsenberg, 2003). It follows therefore that not only are new adjuvants needed, but these must be engineered to stimulate the correct PRR on the optimal cell type. The most clinically relevant illustration of the benefits of adding PRR agonists to increase adjuvant activity is GSK's adjuvant AS 04, which incorporates the TLR4 agonist monophosphoryl lipid A (MPLA) to alum (Didierlaurent et al., 2009). The human papilloma virus virus-like particle vaccine Cervarix is formulated with AS 04, which may account for its apparent increased potency compared to Gardasil, a similar VLP vaccine that uses alum without MPLA (Einstein et al., 2009). Interestingly MPLA appears to preferentially activate TLR4 signalling through the TRIF:TRAM pathway and not Mal:MYD88 (Mata-Haro et al., 2007). 
Without doubt the role of synthetic PRR-specific agonists as additives to vaccine adjuvants will become a developing and increasingly important field of research. Of course given the potent immunostimulatory properties of PRR agonists care will have to be taken to avoid potentially damaging, or fatal, systemic effects.

\subsubsection{TLR agonists in adjuvants}

As well as the benefits of adding MPLA to alum, a wide range of TLR agonists have been shown to promote immunity when added to antigens. These include TLR2-stimulatory lipopeptides such as MALP-2, the TLR5 agonist flagellin, TLR9-stimulatory CpG oligos, small molecule TLR7/8 agonists such as imidazoquinolines, the TLR3 agonist polyI:C and various modified RNA and DNA oligos that can act on TLR3,7 and/or 9. TLR triggering, such as by $\mathrm{CpG}$ oligos or imidazoquinolines, frequently promotes Th1 responses (Schnare et al., 2001), but there are a number of exceptions, including Th2 induction by lipopeptides and flagellin that trigger TLR2 and TLR5 respectively (Didierlaurent et al., 2004; Redecke et al., 2004). It has become clear that for maximal potency and benefits the TLR agonist requires linking in some way with the target antigen. For example, agonists have been covalently coupled to proteins; synthesized directly in combination with the TLR agonist; or antigen and PRR agonists can be immobilised together on a particulate scaffold. The importance of linking PRR agonists with antigen was demonstrated in a recent study where peptides were co-encapsulated with a TLR9 agonist using the biodegradeable polymer PLGA; only when antigen and $\mathrm{CpG}$ oligonucleotides were present in the same PLGA microsphere was an effective cytotoxic T cell response achieved (Schlosser et al., 2008).

\subsubsection{Combinatorial PRR triggering}

Just as most microbes contain a number of different PAMPs and trigger several PRR, there is a need to combine PRR triggers in adjuvants for synergistic effects, and to closely mimic pathogens. The best studied example of synergistic PRR signaling comes from the activation of TLR2 combined with signaling through the CLEC dectin-1 (Gantner et al., 2003; Underhill, 2007). PRR signaling also synergises with other proinflammatory pathways. Major examples include the interplay between $\mathrm{T}$ cell feedback signals through CD40 expressed on PRR-stimulated DC (Schulz et al., 2000), and the requirement of NALP3 for maximal response to various PRR including vaccinia (Delaloye et al., 2009).

\section{Conclusions}

Without doubt PRRs have a crucial role to play in the detection of, and response to, infectious diseases. The repertoire and versatility of the host in detecting PAMPS and associated danger signals is outstanding and continually expanding. In fact this is only matched by the steps taken by pathogens to circumvent these defence strategies. As research progresses in this field it is becoming increasingly apparent that although the outputs of PRR activation are broadly similar - i.e. pro-inflammatory cytokine induction, caspase-1 processing and IFN induction - the mechanisms by which this takes place are actually remarkably subtle. There is significant crosstalk between TLR, NLR, CLR and RLR signalling pathways. In addition, there is clear redundancy in function between different receptors that helps to ensure appropriate recognition of, and response to, pathogens. The 
complexity of the signalling crosstalk is only likely to increase as our understanding of these systems improves.

Another key area in which PRR recognition of infectious diseases is becoming increasingly important is the development of adaptive immunity. The innate response seems to play a vital role in directing the nature of the adaptive response, particularly in relation to driving development of particular T-cell subsets. This is a rapidly developing area and the search for rational vaccine design makes it critical to infectious disease research. The study of the cellular and molecular biology of PRRs has provided a strong theoretical and experimental foundation for the rational design of vaccine adjuvants. This new understanding must be harnessed to provide a complete toolkit for custom adjuvant design. However, in common with other biomedical disciplines, clinical uptake typically lags behind scientific discovery and we wait to see how best this knowledge can be transferred to provide clinical benefit.

\section{Acknowledgements}

The work in our group is funded by The Wellcome Trust (TPM; WT0805090MA) and the Biotechnology and Biological Sciences Research Council (TPM; RG52820). We thank David Dunne for the images of Schistosoma eggs, larvae and adult worms used in Figure 9.

\section{References}

Alexopoulou, L., Thomas, V., Schnare, M., Lobet, Y., Anguita, J., Schoen, R.T., Medzhitov, R., Fikrig, E., and Flavell, R.A. (2002). Hyporesponsiveness to vaccination with Borrelia burgdorferi OspA in humans and in TLR1- and TLR2-deficient mice. Nat Med 8, 878-884.

Andersen-Nissen, E., Smith, K.D., Strobe, K.L., Barrett, S.L., Cookson, B.T., Logan, S.M., and Aderem, A. (2005). Evasion of Toll-like receptor 5 by flagellated bacteria. Proc Natl Acad Sci U S A 102, 9247-9252.

Bi, L., Gojestani, S., Wu, W., Hsu, Y.M., Zhu, J., Ariizumi, K., and Lin, X. (2010). CARD9 mediates dectin-2-induced IkappaBalpha kinase ubiquitination leading to activation of NF-kappaB in response to stimulation by the hyphal form of Candida albicans. J Biol Chem 285, 25969-25977.

Bowie, A., Kiss-Toth, E., Symons, J.A., Smith, G.L., Dower, S.K., and O'Neill, L.A. (2000). A46R and A52R from vaccinia virus are antagonists of host IL-1 and toll-like receptor signaling. Proc Natl Acad Sci U S A 97, 10162-10167.

Bowie, A.G., and Unterholzner, L. (2008). Viral evasion and subversion of patternrecognition receptor signalling. Nat Rev Immunol 8, 911-922.

Cheng, S.C., van de Veerdonk, F.L., Lenardon, M., Stoffels, M., Plantinga, T., Smeekens, S., Rizzetto, L., Mukaremera, L., Preechasuth, K., Cavalieri, D., et al. (2011). The dectin1/inflammasome pathway is responsible for the induction of protective T-helper 17 responses that discriminate between yeasts and hyphae of Candida albicans. J Leukoc Biol 90, 357-366.

Cirl, C., Wieser, A., Yadav, M., Duerr, S., Schubert, S., Fischer, H., Stappert, D., Wantia, N., Rodriguez, N., Wagner, H., et al. (2008). Subversion of Toll-like receptor signaling by a unique family of bacterial Toll/interleukin-1 receptor domain-containing proteins. Nat Med 14, 399-406. 
Coburn, B., Grassl, G.A., and Finlay, B.B. (2007). Salmonella, the host and disease: a brief review. Immunol Cell Biol 85, 112-118.

Davis, B.K., Wen, H., and Ting, J.P. (2011). The inflammasome NLRs in immunity, inflammation, and associated diseases. Annu Rev Immunol 29, 707-735.

De Gregorio, E., D'Oro, U., and Wack, A. (2009). Immunology of TLR-independent vaccine adjuvants. Curr Opin Immunol 21, 339-345.

Delaloye, J., Roger, T., Steiner-Tardivel, Q.G., Le Roy, D., Knaup Reymond, M., Akira, S., Petrilli, V., Gomez, C.E., Perdiguero, B., Tschopp, J., et al. (2009). Innate immune sensing of modified vaccinia virus Ankara (MVA) is mediated by TLR2-TLR6, MDA-5 and the NALP3 inflammasome. PLoS Pathog 5, e1000480.

Didierlaurent, A., Ferrero, I., Otten, L.A., Dubois, B., Reinhardt, M., Carlsen, H., Blomhoff, R., Akira, S., Kraehenbuhl, J.P., and Sirard, J.C. (2004). Flagellin promotes myeloid differentiation factor 88-dependent development of Th2-type response. J Immunol 172, 6922-6930.

Didierlaurent, A.M., Morel, S., Lockman, L., Giannini, S.L., Bisteau, M., Carlsen, H., Kielland, A., Vosters, O., Vanderheyde, N., Schiavetti, F., et al. (2009). AS04, an aluminum salt- and TLR4 agonist-based adjuvant system, induces a transient localized innate immune response leading to enhanced adaptive immunity. J Immunol 183, 6186-6197.

Diebold, S.S., Kaisho, T., Hemmi, H., Akira, S., and Reis e Sousa, C. (2004). Innate antiviral responses by means of TLR7-mediated recognition of single-stranded RNA. Science 303, 1529-1531.

Dougan, G., John, V., Palmer, S., and Mastroeni, P. (2011). Immunity to salmonellosis. Immunol Rev 240, 196-210.

Einstein, M.H., Baron, M., Levin, M.J., Chatterjee, A., Edwards, R.P., Zepp, F., Carletti, I., Dessy, F.J., Trofa, A.F., Schuind, A., et al. (2009). Comparison of the immunogenicity and safety of Cervarix and Gardasil human papillomavirus (HPV) cervical cancer vaccines in healthy women aged 18-45 years. Hum Vaccin 5, 705719.

Esteban, A., Popp, M.W., Vyas, V.K., Strijbis, K., Ploegh, H.L., and Fink, G.R. (2011). Fungal recognition is mediated by the association of dectin-1 and galectin-3 in macrophages. Proceedings of the National Academy of Sciences 108, 14270-14275.

Ferwerda, B., Alonso, S., Banahan, K., McCall, M.B., Giamarellos-Bourboulis, E.J., Ramakers, B.P., Mouktaroudi, M., Fain, P.R., Izagirre, N., Syafruddin, D., et al. (2009a). Functional and genetic evidence that the Mal/TIRAP allele variant 180L has been selected by providing protection against septic shock. Proc Natl Acad Sci U S A 106, 10272-10277.

Ferwerda, B., Ferwerda, G., Plantinga, T.S., Willment, J.A., van Spriel, A.B., Venselaar, H., Elbers, C.C., Johnson, M.D., Cambi, A., Huysamen, C., et al. (2009b). Human dectin1 deficiency and mucocutaneous fungal infections. N Engl J Med 361, 1760-1767.

Franchi, L., Amer, A., Body-Malapel, M., Kanneganti, T.D., Ozoren, N., Jagirdar, R., Inohara, N., Vandenabeele, P., Bertin, J., Coyle, A., et al. (2006). Cytosolic flagellin requires Ipaf for activation of caspase-1 and interleukin 1beta in salmonella-infected macrophages. Nat Immunol 7, 576-582. 
Franchi, L., and Nunez, G. (2008). The Nlrp3 inflammasome is critical for aluminium hydroxide-mediated IL-1beta secretion but dispensable for adjuvant activity. Eur J Immunol 38, 2085-2089.

Fransen, F., Stenger, R.M., Poelen, M.C., van Dijken, H.H., Kuipers, B., Boog, C.J., van Putten, J.P., van Els, C.A., and van der Ley, P. (2010). Differential effect of TLR2 and TLR4 on the immune response after immunization with a vaccine against Neisseria meningitidis or Bordetella pertussis. PLoS One 5, e15692.

Fremond, C.M., Yeremeev, V., Nicolle, D.M., Jacobs, M., Quesniaux, V.F., and Ryffel, B. (2004). Fatal Mycobacterium tuberculosis infection despite adaptive immune response in the absence of MyD88. J Clin Invest 114, 1790-1799.

Gack, M.U., Shin, Y.C., Joo, C.H., Urano, T., Liang, C., Sun, L., Takeuchi, O., Akira, S., Chen, Z., Inoue, S., et al. (2007). TRIM25 RING-finger E3 ubiquitin ligase is essential for RIG-I-mediated antiviral activity. Nature 446, 916-920.

Gantner, B.N., Simmons, R.M., Canavera, S.J., Akira, S., and Underhill, D.M. (2003). Collaborative induction of inflammatory responses by dectin-1 and Toll-like receptor 2. J Exp Med 197, 1107-1117.

Gavin, A.L., Hoebe, K., Duong, B., Ota, T., Martin, C., Beutler, B., and Nemazee, D. (2006). Adjuvant-enhanced antibody responses in the absence of toll-like receptor signaling. Science 314, 1936-1938.

Geeraedts, F., Goutagny, N., Hornung, V., Severa, M., de Haan, A., Pool, J., Wilschut, J., Fitzgerald, K.A., and Huckriede, A. (2008). Superior immunogenicity of inactivated whole virus $\mathrm{H} 5 \mathrm{~N} 1$ influenza vaccine is primarily controlled by Toll-like receptor signalling. PLoS Pathog 4, e1000138.

Gewirtz, A.T., Navas, T.A., Lyons, S., Godowski, P.J., and Madara, J.L. (2001). Cutting edge: bacterial flagellin activates basolaterally expressed TLR5 to induce epithelial proinflammatory gene expression. J Immunol 167, 1882-1885.

Gringhuis, S.I., Wevers, B.A., Kaptein, T.M., van Capel, T.M., Theelen, B., Boekhout, T., de Jong, E.C., and Geijtenbeek, T.B. (2011). Selective C-Rel activation via Malt1 controls anti-fungal $\mathrm{T}(\mathrm{H})-17$ immunity by dectin-1 and dectin-2. PLoS Pathog 7, e1001259.

Guillot, L., Le Goffic, R., Bloch, S., Escriou, N., Akira, S., Chignard, M., and Si-Tahar, M. (2005). Involvement of toll-like receptor 3 in the immune response of lung epithelial cells to double-stranded RNA and influenza A virus. J Biol Chem 280, 5571-5580.

Hajishengallis, G., and Lambris, J.D. (2011). Microbial manipulation of receptor crosstalk in innate immunity. Nat Rev Immunol 11, 187-200.

Hayashi, F., Smith, K.D., Ozinsky, A., Hawn, T.R., Yi, E.C., Goodlett, D.R., Eng, J.K., Akira, S., Underhill, D.M., and Aderem, A. (2001). The innate immune response to bacterial flagellin is mediated by Toll-like receptor 5 . Nature 410, 1099-1103.

Hersh, D., Monack, D.M., Smith, M.R., Ghori, N., Falkow, S., and Zychlinsky, A. (1999). The Salmonella invasin SipB induces macrophage apoptosis by binding to caspase-1. Proc Natl Acad Sci U S A 96, 2396-2401.

Ichinohe, T. (2010). Respective roles of TLR, RIG-I and NLRP3 in influenza virus infection and immunity: impact on vaccine design. Expert Rev Vaccines 9, 1315-1324. 
Iwasaki, A., and Medzhitov, R. (2004). Toll-like receptor control of the adaptive immune responses. Nat Immunol 5, 987-995.

Kanneganti, T.-D. (2010). Central roles of NLRs and inflammasomes in viral infection. Nat Rev Immunol 10, 688-698.

Kapsenberg, M.L. (2003). Dendritic-cell control of pathogen-driven T-cell polarization. Nat Rev Immunol 3, 984-993.

Kawai, T., and Akira, S. (2011). Toll-like receptors and their crosstalk with other innate receptors in infection and immunity. Immunity 34, 637-650.

Kenny, E.F., Talbot, S., Gong, M., Golenbock, D.T., Bryant, C.E., and O'Neill, L.A.J. (2009). MyD88 Adaptor-Like Is Not Essential for TLR2 Signaling and Inhibits Signaling by TLR3. J Immunol 183, 3642-3651.

Khor, C.C., Chapman, S.J., Vannberg, F.O., Dunne, A., Murphy, C., Ling, E.Y., Frodsham, A.J., Walley, A.J., Kyrieleis, O., Khan, A., et al. (2007). A Mal functional variant is associated with protection against invasive pneumococcal disease, bacteremia, malaria and tuberculosis. Nat Genet 39, 523-528.

LeibundGut-Landmann, S., Gross, O., Robinson, M.J., Osorio, F., Slack, E.C., Tsoni, S.V., Schweighoffer, E., Tybulewicz, V., Brown, G.D., Ruland, J., et al. (2007). Syk- and CARD9-dependent coupling of innate immunity to the induction of $\mathrm{T}$ helper cells that produce interleukin 17. Nat Immunol 8, 630-638.

Leroux-Roels, G. (2010). Unmet needs in modern vaccinology: adjuvants to improve the immune response. Vaccine 28 Suppl 3, C25-36.

Li, H., Willingham, S.B., Ting, J.P., and Re, F. (2008). Cutting edge: inflammasome activation by alum and alum's adjuvant effect are mediated by NLRP3. J Immunol 181, 17-21.

Loo, Y.M., and Gale, M., Jr. (2011). Immune signaling by RIG-I-like receptors. Immunity 34, 680-692.

Lu, Y., Wambach, M., Katze, M.G., and Krug, R.M. (1995). Binding of the influenza virus NS1 protein to double-stranded RNA inhibits the activation of the protein kinase that phosphorylates the elF-2 translation initiation factor. Virology 214, 222-228.

Lund, J.M., Alexopoulou, L., Sato, A., Karow, M., Adams, N.C., Gale, N.W., Iwasaki, A., and Flavell, R.A. (2004). Recognition of single-stranded RNA viruses by Toll-like receptor 7. Proc Natl Acad Sci U S A 101, 5598-5603.

Martinez, J., Huang, X., and Yang, Y. (2010). Toll-like receptor 8-mediated activation of murine plasmacytoid dendritic cells by vaccinia viral DNA. Proc Natl Acad Sci U S A 107, 6442-6447.

Mata-Haro, V., Cekic, C., Martin, M., Chilton, P.M., Casella, C.R., and Mitchell, T.C. (2007). The vaccine adjuvant monophosphoryl lipid A as a TRIF-biased agonist of TLR4. Science 316, 1628-1632.

Mbow, M.L., De Gregorio, E., Valiante, N.M., and Rappuoli, R. (2010). New adjuvants for human vaccines. Curr Opin Immunol 22, 411-416.

McKee, A.S., Munks, M.W., MacLeod, M.K., Fleenor, C.J., Van Rooijen, N., Kappler, J.W., and Marrack, P. (2009). Alum induces innate immune responses through macrophage and mast cell sensors, but these sensors are not required for alum to act as an adjuvant for specific immunity. J Immunol 183, 4403-4414. 
Miao, E.A., Mao, D.P., Yudkovsky, N., Bonneau, R., Lorang, C.G., Warren, S.E., Leaf, I.A., and Aderem, A. (2010). Innate immune detection of the type III secretion apparatus through the NLRC4 inflammasome. Proceedings of the National Academy of Sciences 107, 3076-3080.

Monie, T.P., Bryant, C.E., and Gay, N.J. (2009). Activating immunity: lessons from the TLRs and NLRs. Trends Biochem Sci 34, 553-561.

Nemazee, D., Gavin, A., Hoebe, K., and Beutler, B. (2006). Immunology: Toll-like receptors and antibody responses. Nature 441, E4; discussion E4.

Osorio, F., and Reis e Sousa, C. (2011). Myeloid C-type lectin receptors in pathogen recognition and host defense. Immunity 34, 651-664.

Perry, A.K., Chen, G., Zheng, D., Tang, H., and Cheng, G. (2005). The host type I interferon response to viral and bacterial infections. Cell Res 15, 407-422.

Pichlmair, A., Schulz, O., Tan, C.P., Naslund, T.I., Liljestrom, P., Weber, F., and Reis e Sousa, C. (2006). RIG-I-mediated antiviral responses to single-stranded RNA bearing 5'phosphates. Science 314, 997-1001.

Redecke, V., Häcker, H., Datta, S.K., Fermin, A., Pitha, P.M., Broide, D.H., and Raz, E. (2004). Cutting Edge: Activation of Toll-Like Receptor 2 Induces a Th2 Immune Response and Promotes Experimental Asthma. The Journal of Immunology 172, 2739-2743.

Reed, S.G., Bertholet, S., Coler, R.N., and Friede, M. (2009). New horizons in adjuvants for vaccine development. Trends Immunol 30, 23-32.

Ritter, M., Gross, O., Kays, S., Ruland, J., Nimmerjahn, F., Saijo, S., Tschopp, J., Layland, L.E., and Prazeres da Costa, C. (2010). Schistosoma mansoni triggers Dectin-2, which activates the Nlrp3 inflammasome and alters adaptive immune responses. Proc Natl Acad Sci U S A 107, 20459-20464.

Roy, C.R., and Zamboni, D.S. (2006). Cytosolic detection of flagellin: a deadly twist. Nat Immunol 7, 549-551.

Sabbah, A., Chang, T.H., Harnack, R., Frohlich, V., Tominaga, K., Dube, P.H., Xiang, Y., and Bose, S. (2009). Activation of innate immune antiviral responses by Nod2. Nat Immunol 10, 1073-1080.

Saijo, S., Ikeda, S., Yamabe, K., Kakuta, S., Ishigame, H., Akitsu, A., Fujikado, N., Kusaka, T., Kubo, S., Chung, S.H., et al. (2010). Dectin-2 recognition of alpha-mannans and induction of Th17 cell differentiation is essential for host defense against Candida albicans. Immunity 32, 681-691.

Schlosser, E., Mueller, M., Fischer, S., Basta, S., Busch, D.H., Gander, B., and Groettrup, M. (2008). TLR ligands and antigen need to be coencapsulated into the same biodegradable microsphere for the generation of potent cytotoxic $\mathrm{T}$ lymphocyte responses. Vaccine 26, 1626-1637.

Schmitz, N., Kurrer, M., Bachmann, M.F., and Kopf, M. (2005). Interleukin-1 is responsible for acute lung immunopathology but increases survival of respiratory influenza virus infection. J Virol 79, 6441-6448.

Schnare, M., Barton, G.M., Holt, A.C., Takeda, K., Akira, S., and Medzhitov, R. (2001). Tolllike receptors control activation of adaptive immune responses. Nat Immunol 2, 947-950. 
Schulz, O., Edwards, A.D., Schito, M., Aliberti, J., Manickasingham, S., Sher, A., and Reis e Sousa, C. (2000). CD40 triggering of heterodimeric IL-12 p70 production by dendritic cells in vivo requires a microbial priming signal. Immunity 13, 453-462.

Smeekens, S.P., van de Veerdonk, F.L., van der Meer, J.W., Kullberg, B.J., Joosten, L.A., and Netea, M.G. (2010). The Candida Th17 response is dependent on mannan- and beta-glucan-induced prostaglandin E2. Int Immunol 22, 889-895.

Smith, K.D., Andersen-Nissen, E., Hayashi, F., Strobe, K., Bergman, M.A., Barrett, S.L., Cookson, B.T., and Aderem, A. (2003). Toll-like receptor 5 recognizes a conserved site on flagellin required for protofilament formation and bacterial motility. Nat Immunol 4, 1247-1253.

Spiller, S., Elson, G., Ferstl, R., Dreher, S., Mueller, T., Freudenberg, M., Daubeuf, B., Wagner, H., and Kirschning, C.J. (2008). TLR4-induced IFN-gamma production increases TLR2 sensitivity and drives Gram-negative sepsis in mice. J Exp Med 205, 1747-1754.

Stewart, C.R., Stuart, L.M., Wilkinson, K., van Gils, J.M., Deng, J., Halle, A., Rayner, K.J., Boyer, L., Zhong, R., Frazier, W.A., et al. (2009). CD36 ligands promote sterile inflammation through assembly of a Toll-like receptor 4 and 6 heterodimer. Nat Immunol 11, 155-161.

Subramanian, N., and Qadri, A. (2006). Lysophospholipid sensing triggers secretion of flagellin from pathogenic salmonella. Nat Immunol 7, 583-589.

Suram, S., Gangelhoff, T.A., Taylor, P.R., Rosas, M., Brown, G.D., Bonventre, J.V., Akira, S., Uematsu, S., Williams, D.L., Murphy, R.C., et al. (2010). Pathways regulating cytosolic phospholipase A2 activation and eicosanoid production in macrophages by Candida albicans. J Biol Chem 285, 30676-30685.

Takahara, K., Tokieda, S., Nagaoka, K., Takeda, T., Kimura, Y., and Inaba, K. (2011). C-type lectin SIGNR1 enhances cellular oxidative burst response against C. albicans in cooperation with Dectin-1. Eur J Immunol 41, 1435-1444.

Talbot, S., Tötemeyer, S., Yamamoto, M., Akira, S., Hughes, K., Gray, D., Barr, T., Mastroeni, P., Maskell, D.J., and Bryant, C.E. (2009). Toll-like receptor 4 signalling through MyD88 is essential to control $<\mathrm{i}>$ Salmonella enterica $</ \mathrm{i}>$ serovar Typhimurium infection, but not for the initiation of bacterial clearance. Immunology 128, 472-483.

Underhill, D.M. (2007). Collaboration between the innate immune receptors dectin-1, TLRs, and Nods. Immunol Rev 219, 75-87.

Van der Graaf, C.A., Netea, M.G., Morre, S.A., Den Heijer, M., Verweij, P.E., Van der Meer, J.W., and Kullberg, B.J. (2006). Toll-like receptor 4 Asp299Gly/Thr399Ile polymorphisms are a risk factor for Candida bloodstream infection. Eur Cytokine Netw 17, 29-34.

van Stijn, C.M., Meyer, S., van den Broek, M., Bruijns, S.C., van Kooyk, Y., Geyer, R., and van Die, I. (2010). Schistosoma mansoni worm glycolipids induce an inflammatory phenotype in human dendritic cells by cooperation of TLR4 and DC-SIGN. Mol Immunol 47, 1544-1552.

Wang, J.P., Liu, P., Latz, E., Golenbock, D.T., Finberg, R.W., and Libraty, D.H. (2006). Flavivirus activation of plasmacytoid dendritic cells delineates key elements of TLR7 signaling beyond endosomal recognition. J Immunol 177, 7114-7121. 
Werninghaus, K., Babiak, A., Gross, O., Holscher, C., Dietrich, H., Agger, E.M., Mages, J., Mocsai, A., Schoenen, H., Finger, K., et al. (2009). Adjuvanticity of a synthetic cord factor analogue for subunit Mycobacterium tuberculosis vaccination requires FcRgamma-Syk-Card9-dependent innate immune activation. J Exp Med 206, 89-97.

Williams, A., Flavell, R.A., and Eisenbarth, S.C. (2010). The role of NOD-like Receptors in shaping adaptive immunity. Curr Opin Immunol 22, 34-40.

Woehrle, T., Du, W., Goetz, A., Hsu, H.Y., Joos, T.O., Weiss, M., Bauer, U., Brueckner, U.B., and Marion Schneider, E. (2008). Pathogen specific cytokine release reveals an effect of TLR2 Arg753Gln during Candida sepsis in humans. Cytokine 41, 322-329.

Zhu, J., Martinez, J., Huang, X., and Yang, Y. (2007). Innate immunity against vaccinia virus is mediated by TLR2 and requires TLR-independent production of IFN-beta. Blood 109, 619-625. 


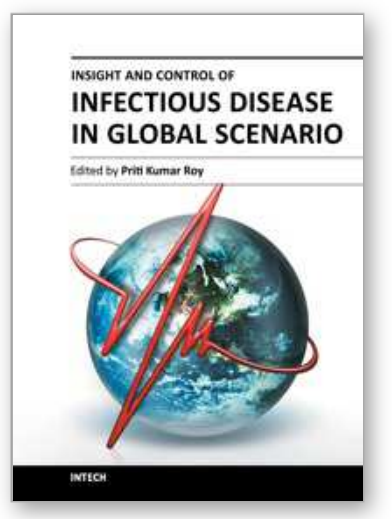

\author{
Insight and Control of Infectious Disease in Global Scenario \\ Edited by Dr. Roy Priti
}

ISBN 978-953-51-0319-6

Hard cover, 442 pages

Publisher InTech

Published online 21, March, 2012

Published in print edition March, 2012

This book is projected as a preliminary manuscript in Infectious Disease. It is undertaken to cover the foremost basic features of the articles. Infectious Disease and analogous phenomenon have been one of the main imperative postwar accomplishments in the world. The book expects to provide its reader, who does not make believe to be a proficient mathematician, an extensive preamble to the field of infectious disease. It may immeasurably assist the Scientists and Research Scholars for continuing their investigate workings on this discipline. Numerous productive and precise illustrated descriptions with a number of analyses have been included. The book offers a smooth and continuing evolution from the principally disease oriented lessons to a logical advance, providing the researchers with a compact groundwork for upcoming studies in this subject.

\title{
How to reference
}

In order to correctly reference this scholarly work, feel free to copy and paste the following:

Ardi Liaunardy Jopeace, Chris B. Howard, Ben L. Murton, Alexander D. Edwards and Tom P. Monie (2012). Pattern Recognition Receptors and Infectious Diseases, Insight and Control of Infectious Disease in Global Scenario, Dr. Roy Priti (Ed.), ISBN: 978-953-51-0319-6, InTech, Available from: http://www.intechopen.com/books/insight-and-control-of-infectious-disease-in-global-scenario/patternrecognition-receptors-and-infectious-disease

\section{INTECH}

open science | open minds

\author{
InTech Europe \\ University Campus STeP Ri \\ Slavka Krautzeka 83/A \\ 51000 Rijeka, Croatia \\ Phone: +385 (51) 770447 \\ Fax: +385 (51) 686166 \\ www.intechopen.com
}

\author{
InTech China \\ Unit 405, Office Block, Hotel Equatorial Shanghai \\ No.65, Yan An Road (West), Shanghai, 200040, China \\ 中国上海市延安西路65号上海国际贵都大饭店办公楼 405 单元 \\ Phone: +86-21-62489820 \\ Fax: +86-21-62489821
}


(C) 2012 The Author(s). Licensee IntechOpen. This is an open access article distributed under the terms of the Creative Commons Attribution 3.0 License, which permits unrestricted use, distribution, and reproduction in any medium, provided the original work is properly cited. 